\title{
Effect of Applying Total Quality Management in Improving the Performance of Al-Waqf of Albr Societies in Saudi Arabia: A theoretical framework for "Deming's Model"
}

Aldwihi, Zakaria Ali Saad*

Islamic Banking \& Finance (IIiBF),

International Islamic University Malaysia,

Selangor

Email: zaldwihi@gmail.com

\section{Azman Bin Mohd. Noor}

Islamic Banking \& Finance (IIiBF),

International Islamic University Malaysia,

Selangor

\section{Ashurov Sharofiddin}

Islamic Banking \& Finance (IIiBF),

International Islamic University Malaysia,

Selangor

Received March, 2020; Accepted May, 2020

Abstract: This study aims to examine the effect of applying Total Quality Management (TQM) on the performance of Al-Waqf of Albr societies in the Kingdom of Saudi Arabia. Such charitable organizations lack transparency and are requested to provide adequate financial disclosure. The lack of internal control and scrutiny negatively affects their performance. The study relies on Deming's 14-Point to assess Waqf quality control and effective management and leadership styles. The analysis is qualitative. Interviews and case studies were used to achieve the research objectives. The study fills the research gap and weighs the impact of TQM application for charitable organizations in Saudi Arabia.

Keywords: Total Quality Management; TQM; Waqf Institutions; Waqf Quality

Type: Research paper
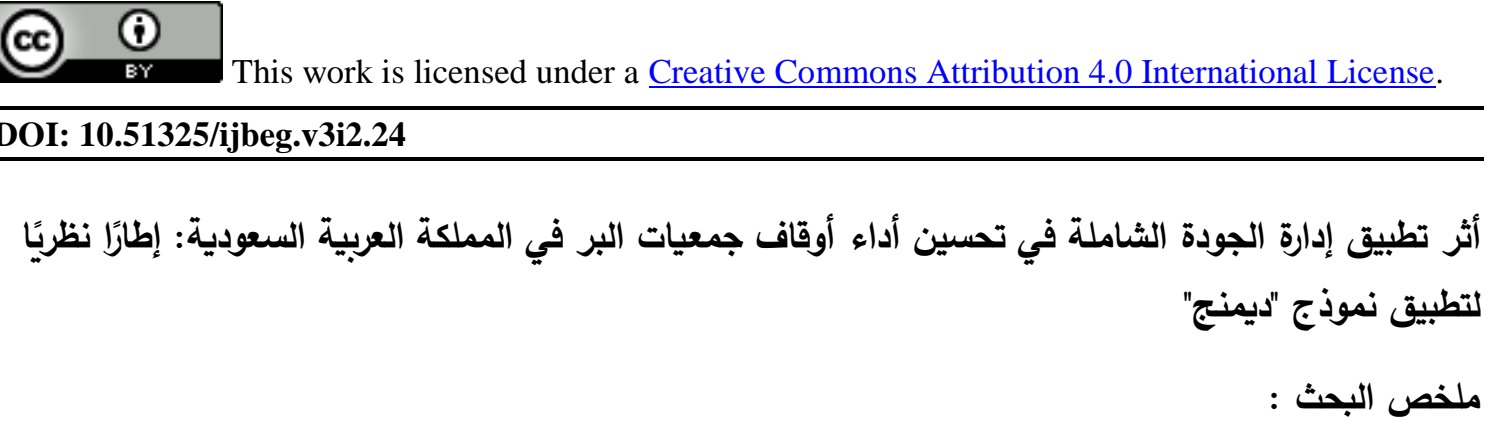
يعد الوقف تشريعاً إسلامياً أصيلاً يستمد مشروعيته من السنة النبوية وتعاليم الإسلام الحكيم كالصدقات والتعاون

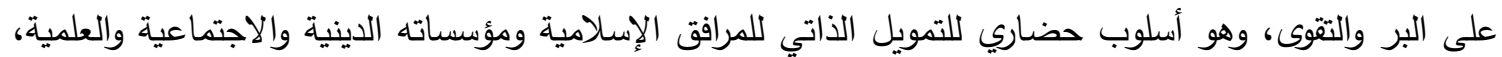

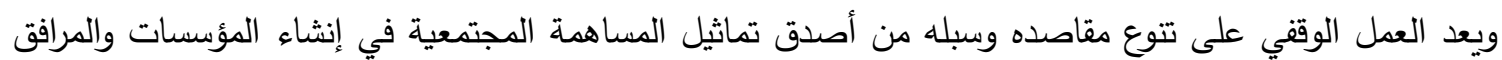

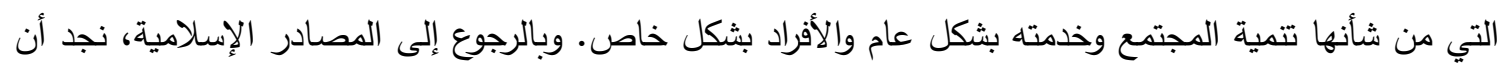

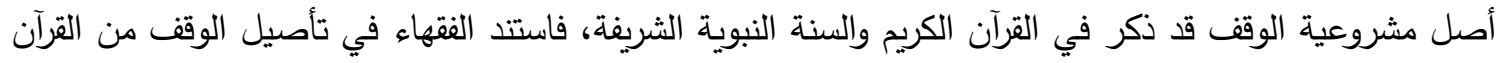

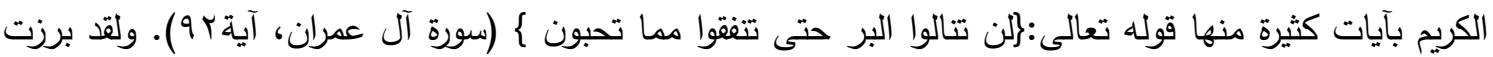
أهمية الوقف في السنة النبوية أيضاً من خلال ما روي عن أبي هريرة (رضي الله عنه)، أن النبي صلى الله عليه وسلم قال: "إذا مات ابن آدم، انقطع عمله إلا من ثلاث: صدقة جارية، أو علم ينتفع به، أو ولا صالح يدعوا لها له (صحيح مسلم). وكما هو معروف، فإن الأوقاف في العصر الحالي يتم إدارتها بصورة مؤسية وبإشراف القطاع الحكومي، أو من

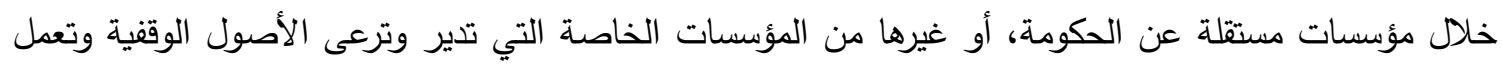

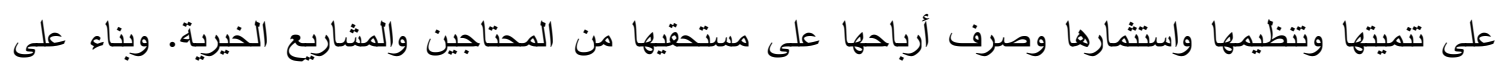
ذلك، فإن العاملين في قطاع الأوقاف الإسلامية ملزمون بالمثابرة والعمل على تحقيق مبادئ إدارة الجودة الثاملة

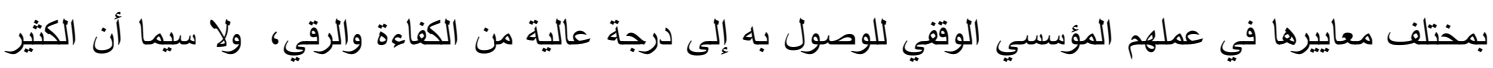

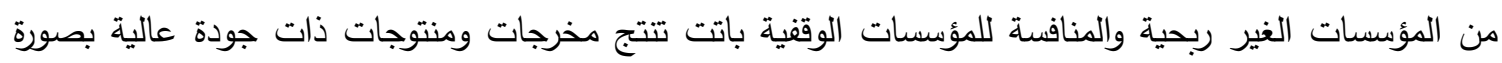
تبين مدى الفجوة والفرق بين عمل هذه المؤسسات و المؤسسات الوقفية في المملكة العربية السعودية التي لا زالت

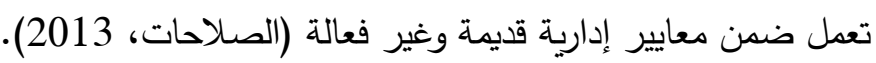
اهتمت الكثير من دول العالم بثكل عام ودول العالم الإسلامي والخليج بثكل خاص بالوقف وإدارته والاهتمام به كالكويت وغيرها كما اهتمت المملكة العربية السعودية أيضاً ممثلة بوزارة الأوقاف، المعروفة حالياً باسم وزارة الثئون

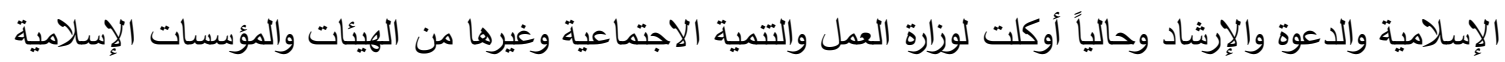
بالأوقاف الإسلامية، وذلك لما للوقف من أهمية كبرى في تتمية المجتمعات وفي الحراك الاجتماعي الفعال الذي ورداتي

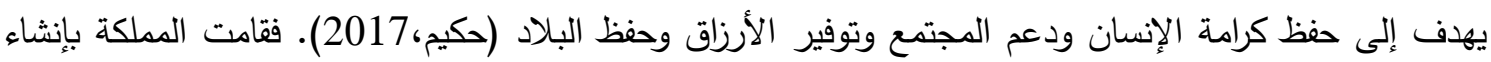

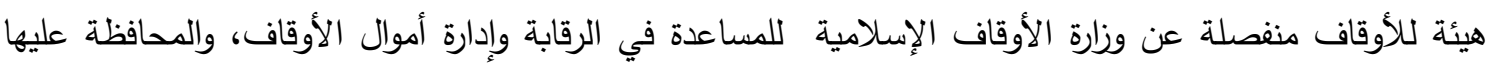

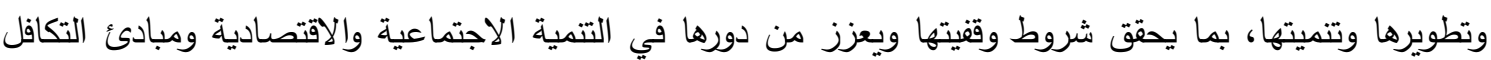

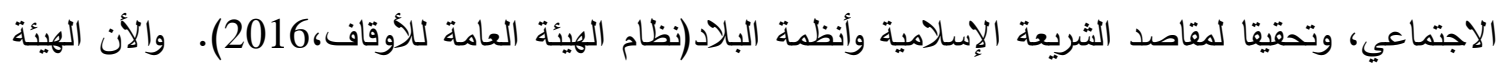
في شراكة مع وزارة العمل والتتمية الاجتماعية من خلال برنامج "استدامة وتمكين" مواكبة مع رؤية المملكة 2030

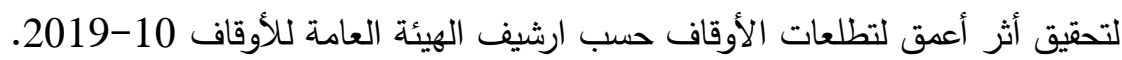
وكان من أبرز توجهات المملكة العربية السعودية في السنوات الأخيرة هو تطبيق مبادئ إدارة الجودة بمفاهيمها الثاملة في وزاراتها ومؤسساتها الحكومية والخاصة على حد سواء، وعلى مختلف التخصصات والمجالات سواء

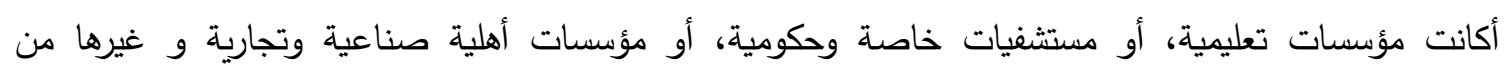
المؤسسات والهيئات؛ لما لها من أهمية بالغة في تتبع وجوه القصور وضمان سلانهة الهات المخرجات قدر المستطاع، والارتقاء بالخدمات والمنتجات، رغبة من البلاد في التغيير للأفضل، ودفع عجلة التطور والتتمية والازدهار (رؤية المملكة العربية السعودية 2030). فالمشكلة تكمن هنا بعدم وجود نظام اداري متكامل للجودة يطبق بالثكل السليم 
في الأوقاف كإدارة الجودة الثاملة والتي بدورها تقوم بحفظ مراقبته وصيانة الوقف بكفاءة وفاعليه للرقي بالقطاع الوقفي وجني ثماره.

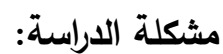

منذ بداية النهضة الصناعية 1940م إلى يومنا هذا وإدارة الجودة تحصد الكثير من الفوائد على مطبقيها فبتطبيق

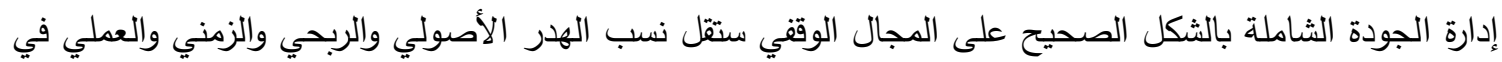
الأوقاف من خلال الاقتتاع التام به وبتوعية العاملين بأهيته وتهيئة البيئة التتظيمية السليمة من التخطيط والرقابة التئي الثاملة والعمل الجماعي المتكاتف المنسق السليم وسيسير حينها الوقف بخطوات ثابتة ومنسقة وقوية إلى طريق النجاح والنمو والازدهار إذ يؤكد الجبير الجودة بدراسة قام بها على عينة تضم 500 شركة أمريكية أن برنامج إدارة الجودة الثاملة لها تأثير ذو دلالة على قدرتهم التنافسية، فالقيم الجوهرية لإقامة الجودة

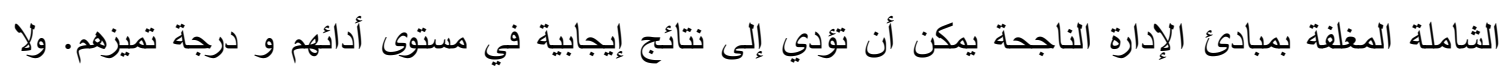
يتحقق التمايز إلا إذا عم الالتزام بالجودة في جميع مستويات الأداء بمعنى المدخلات و المخرجات والعمليات. (بدر ، 2009)

ومن الناحية الأخرى، يكاد لا يخفى على كثيراً من المهتمين بالدراسات الوقفية البناء الضعيف الذي آل إليه الوقف فالف

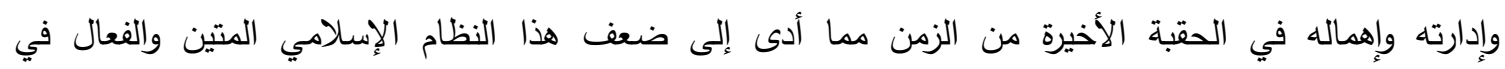
المجتمعات مما يتطلب منا تدعيمه بنظام قوي وفعال ومجرب للنهوض به مراه مرة أخرى كي يخدم البلاد والعباد وهنا استعراض لبعض المعضلات التي جعلت من تطبيق إدارة الجودة الثاملة بالثكل الصحيح ضرورة ملحة كما أثبت

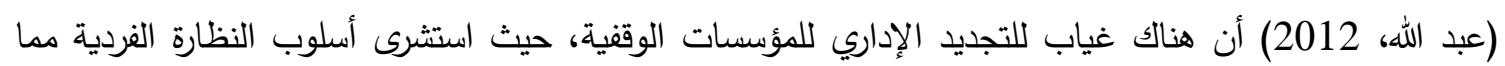

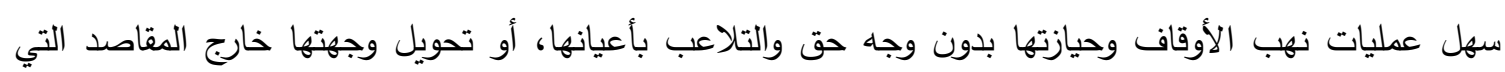

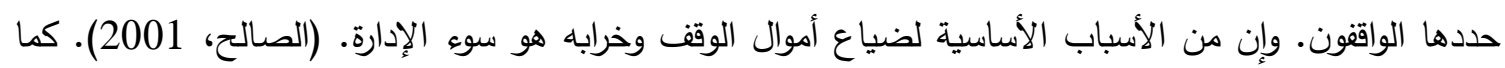

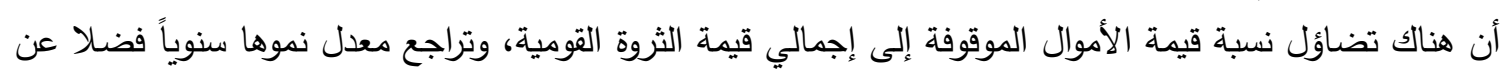

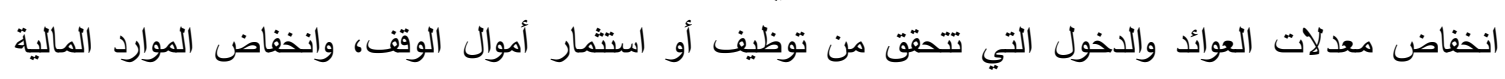

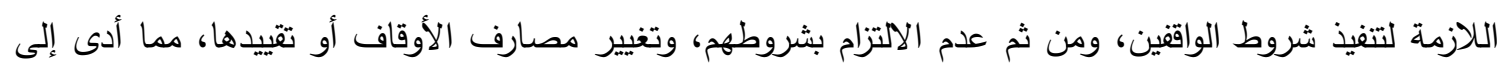
حرمان العديد من الجهات من حقوقها، وتعطلت رسالة الوقف لدرجة التهديد بالقضاء عليها. كما أن هناك إهمال

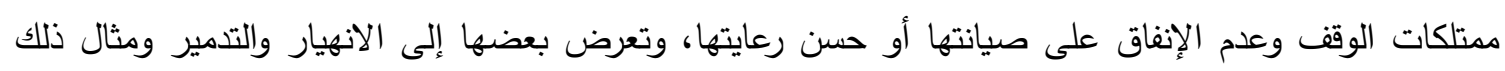

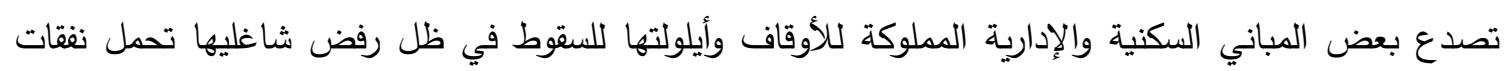

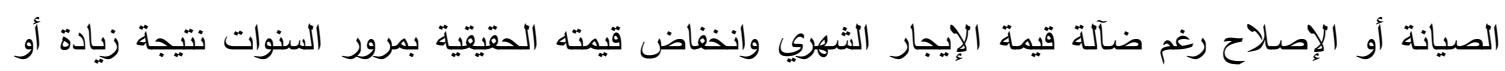
غلاء الأسعار وتآكل قيمة النقود. كما أن تركيز بعض الجهات المشرفة على الوقار الوقف على التوثيق والسجلات فقط

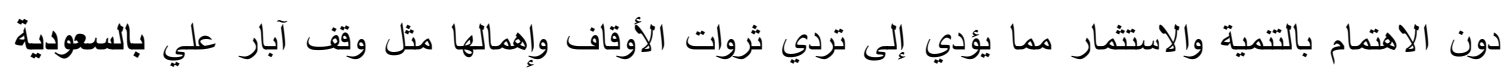

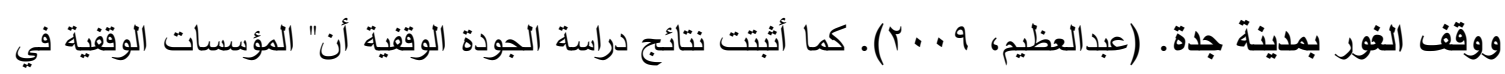
المملكة العربية السعودية بحاجة ملحة إلى جودة وتحسين وضبط لكفاءة الأداء المؤسسي، ولا يتوفر ذلك إلإنها من

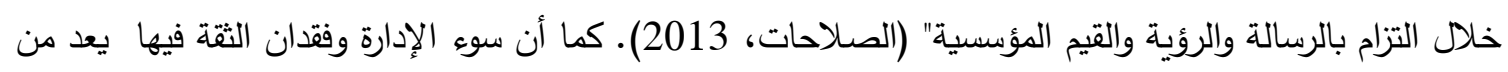

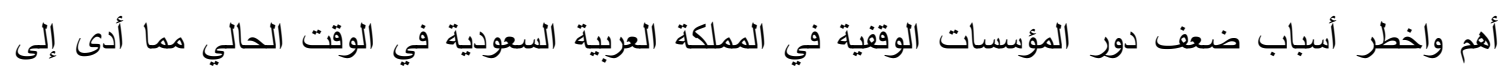

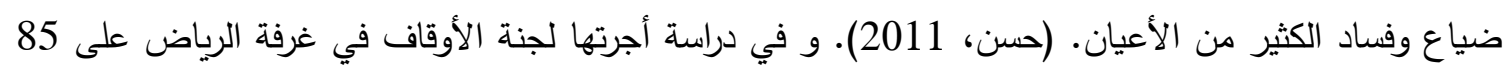

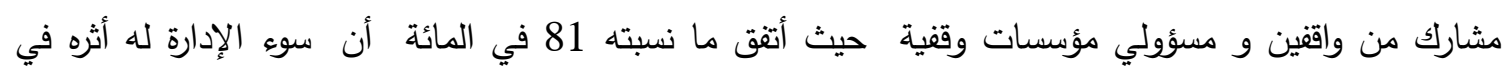


انخفاض عوائد الوقف وأوصت الدراسة بضرورة تفعيل الحوكمة ومجالس الرقابة على الأوقاف. (الثميري، 2016) وأكد نائب رئيس اللجنة الوطنية للأوقاف الثيخ محمد الزامل أن من المهم تأهيل وتدريب القائمين على إدارة الأوقاف وأن من أهم وأكبر معوقات الأوقاف عدم وضوح الأنظمة وضعف الرقافية الرقابة وعدم وجود آلية. (الغنيم، 2016)

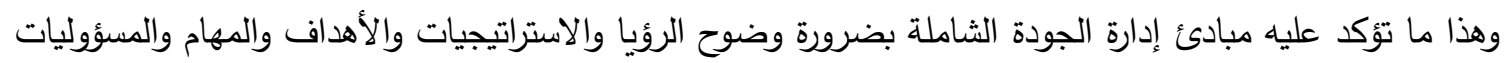
وضرورة التدريب والتوعية مع الرقابة على كل القرارات والإجراءات والعمليات ومراجعتها دورياً وتصحيح الانحرافات.

ونظرًا للنمو والتطور المشهود في مجال الأوقاف في أوساط المجتمات الإسلامية عامة، وفي المملكة العربية

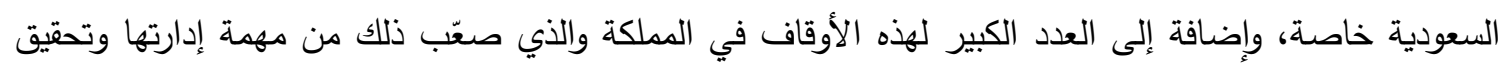

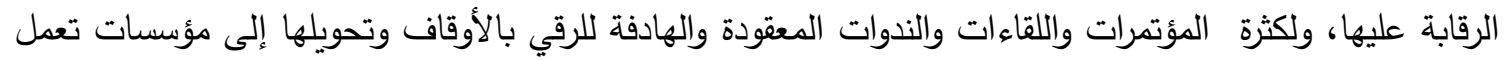

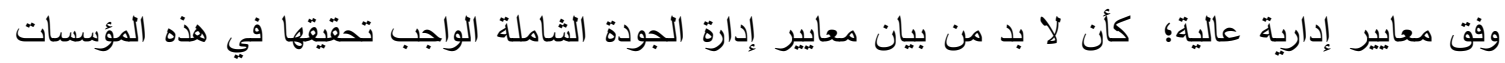

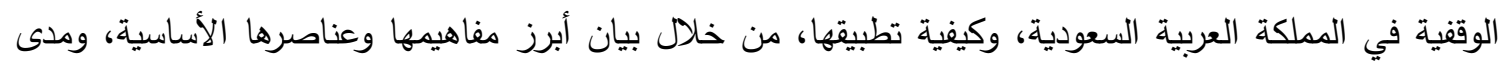
تطبيقها وتفعيلها على المؤسسات بثكل عام، وعلى المؤسسات الوقفية في المملكة العربية السعودية بصورة خاصنة (الصلاحات،2013). وقال راجس الدوسري نائب رئيس لجنة الأوقاف بالغرفة التجارية بالرياض أن قطاع الأوقاف

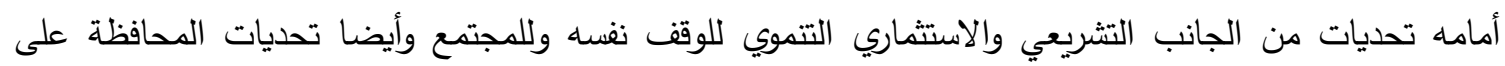
الأصول الوقفية وتحقيق أركان الوقف وأهدافه. (وكالة الأنباء السعودية، 2019) ولا تكون المحافظة على أصول

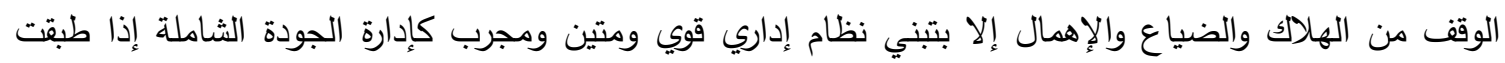

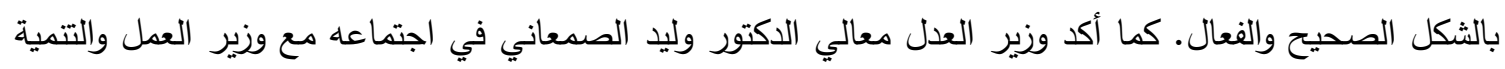
الاجتماعية ومحافظ الهيئة العامة للأوقاف وعدد من الخبراء على "ضرورة ضبط العمل الوقفي بمزيد من اللوائح

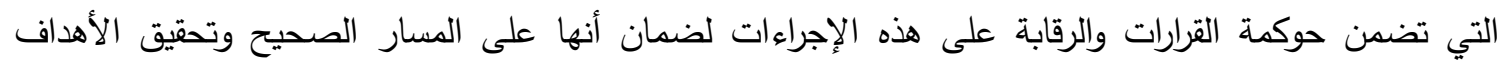
المحددة لها وناقش الاجتماع التحديات والمعوقات التي تواجه الأوقاف في المملكة العربية السعودية وسبل حلهاء

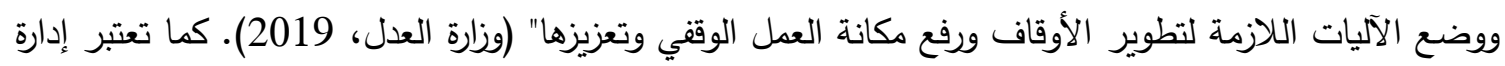
الجودة الثاملة نظام رقابي قوي وفعال ومجرب نجاحه على النطاق الدحلي والدولي ويسعى إلى التطوير والتنمية

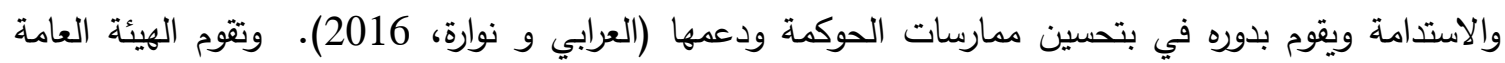

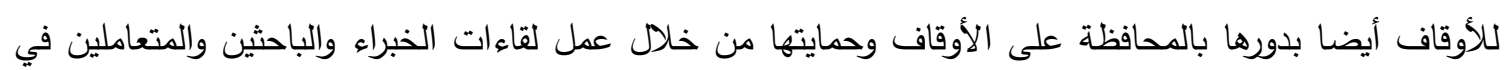

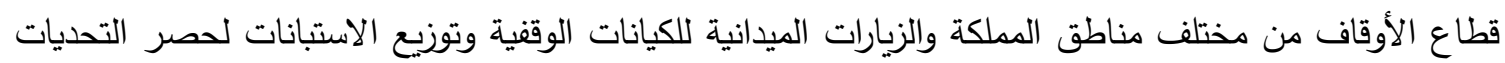
والصعوبات التي تواجه القطاع الوقفي ومحاولة الحد منها ووضع الحلول المدكنة لها من خلال بناء استراتيجية

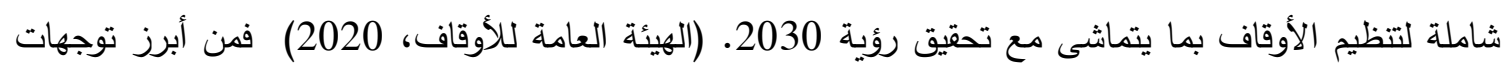
المملكة العربية السعودية في السنوات الأخيرة هو تطبيق مبادئ إدارة الجودة بمفاهيمها الثاملة في وزاراتها ومؤسساتها الحكومية والخاصة على حد سواء، وعلى مختلف التخصصات والمجالات سواء أكانت مؤسسات

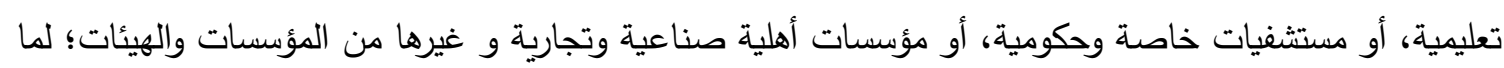
لها من أهمية بالغة في تتبع وجوه القصور وضمان سلامة المخرجات قدر المستطاع، والارتقاء بالخدمات والمنتجات، رغبة من البلاد في التغيير للأفضل، ودفع عجلة التطور والتتمية والازدهار (رؤية لعربية السعودية 


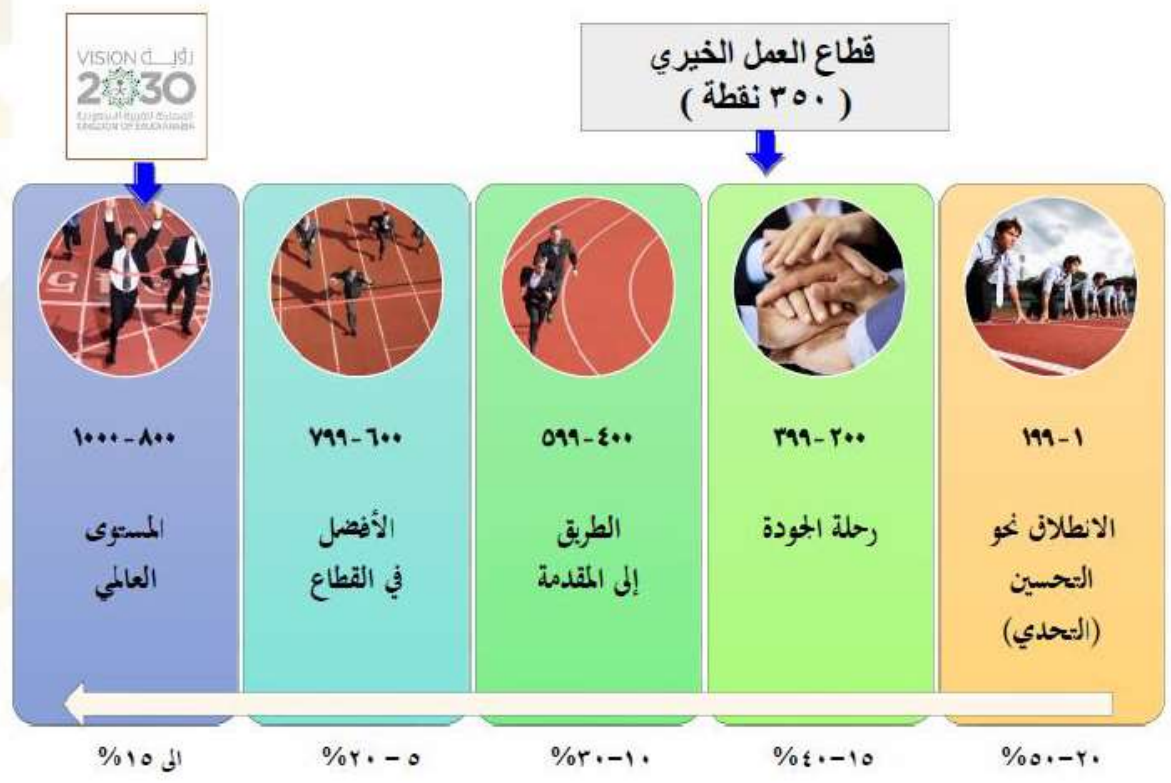

يتضح من تقرير رحلة الجودة لـ (المجلس السعودي للجودة، 2019) أنه لم يتجاوز منتصف الطريق للتميز العالمي

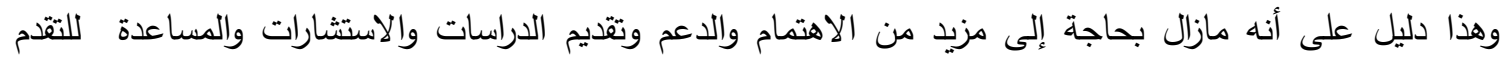

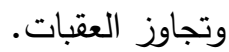

كما اهتمت العديد من الدراسات بإيجاد مفتاح النجاح لمشكلات المؤسسات الغير وقفية بشكل عام في البحث بفاعلية وبكيفية تطبيق مبادئ إدارة الجودة الثاملة على المؤسسات التعليمية والأهلية وغيرها من المؤسسات بمختلف التخصصات بالثكل الصحيح، إلا أن الدراسات التي عنيت بالبحث في كيفية تطبيق هذه المبادئ وقياس مدى تطبيقها على أوقاف جمعيات البر في المملكة العربية السعودية بالثكل الصحيح شحيحة بحسب علم الباحث، الأمر الذي أحدث فجوةً واضحةً وفرقاً واضحاً في الأداء والمخرجات بين هذه المؤسسات ومؤسسات الأوقاف في المملكة العربية السعودية. وكل ما سبق دليل واقعي للحاجة لقياس مدى فاعلية تطبيق إدارة الجودة الثاملة في أوقاف جمعيات البر في المملكة العربية السعودية. وفي ضوه العرض والتحليل السابق يمكن تحديد مشكلة الدراسة في التالي: قياس فاعلية تطبيق إدارة الجودة الثاملة في أوقاف جمعيات البر في المملكة العربية السعودية.

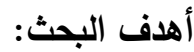

يهدف البحث بشكل رئيسي إلى : قياس فاعلية تطبيق إدارة الجودة الثاملة على أوقاف جمعيات البر في المملكة

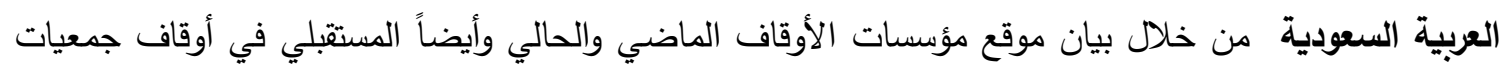

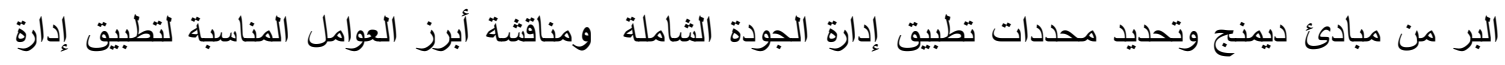


الجودة الثاملة في مؤسسات الأوقاف والتعرف على معوقات وآفاق تطبيق إدارة الجودة الثاملة في مؤسسات الأوقاف في المملكة العربية السعودية.

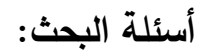

يتمثل سؤال الدراسة الرئيسي في: ما هي فاعلية تطبيق إدارة الجودة الثاملة على أوقاف جمعيات البر في المملكة العربية السعودية؟

ويتفرع من هذا السؤال الرئيسي السابق التساؤلات الفرعية التالية:

1. ما هو الواقع الماضي والحالي والمستقبلي لإدارة الجودة الثاملة في أوقاف جمعيات البر في الممكة العربية السعودية من مبادئ ديمنج؟

2. ما هي محددات تطبيق إدارة الجودة الثاملة في مؤسسات الأوقاف بالمملكة العربية السعودية ؟

3. ما هي العوامل المناسبة لتطبيق إدارة الجودة الثاملة في مؤسسات الأوقاف في المملكة العربية السعودية ؟

4. ما هي المعوقات التي تواجه تطبيق إدارة الجودة الثاملة في مؤسسات الأوقاف في المملكة العربية

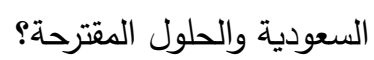

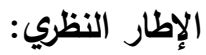

إدارة الجودة الثاملة شكل تعاوني لأداء الأعمال وبتحريك المواهب والقدرات لكل من العاملين والإدارة لتحسين

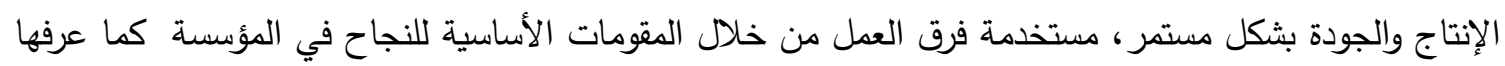

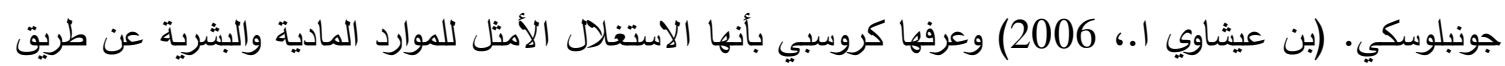

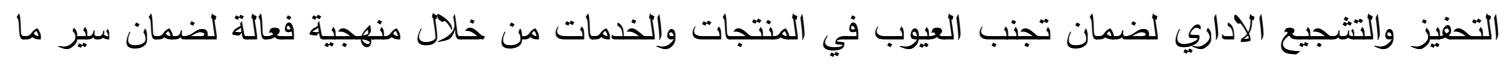

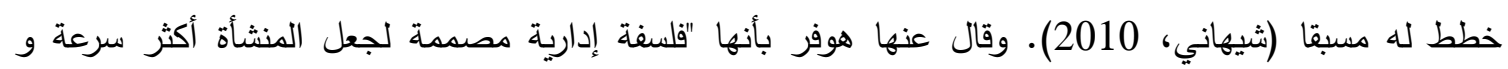
مرونة، بحيث تسهح هذه الطريقة في ظهور نظام متين التركيب يوجه جهد كل موظف إلى كسب ثنقة العهيل، كما تسهم هذه الطريقة في استحداث بيئة تسمح بمشاركة منسوبي المنشأة جميعهم في التخطيط و تتفيذ أساليب التحسين

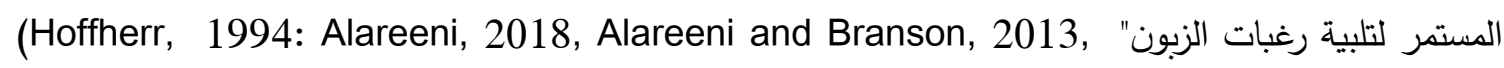
Alareeni, 2019).

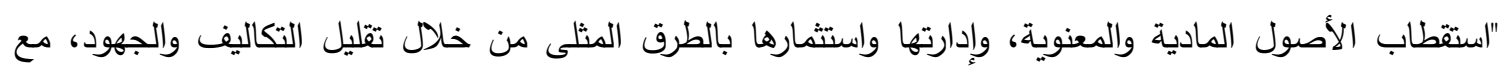

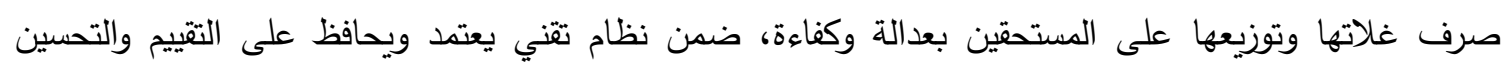

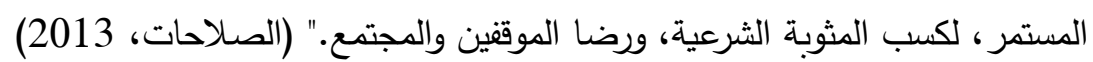

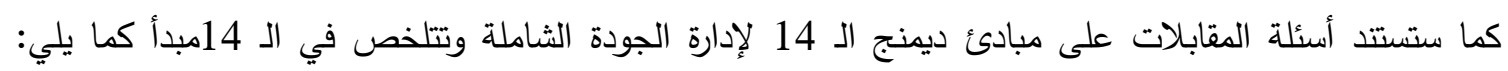
(عقيلي، 2001)

1. تحديد أهداف ثابتة للمنشأة استراتيجية طويلة الأمد من أجل تحسين جودة منتجاتها وخدماتها، وضرورة

نشر تلك الأهداف من خلال رسالتها إلى المهتمين كالمستفيدين من الموظفين وأفراد المجتمع المحلي. 
2. انتهاج فلسفة الجودة يجب أن يمثل قراراً يشترك فيه كل فرد في المؤسسة وإزالة العوائق والحدود التنظيمية بين الموظفين.

3. تبنى هدف الرقابة من كثف الخطأ والمحاسبة، إلى رقابة وقائية تستهدف منع وقوع الخطأ وتقديم الدعم

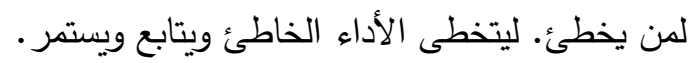
4. عدم بناء قرارات على أساس التكاليف فقط، فالمهم ليس الحلول السهلة، لأنها قد تكون أكثر تكلفة، ولذا ولاء لابد من تغيير فلسفة الشراء التي تعتمد على السعر فقط. 5. تأصيل التدريب المستمر على رأس العمل مع الاعتماد على الطرق الحديثة في التدريب وربطها بخطط تعطي

$$
\text { تحسين الجودة. }
$$

6. التوقف عن استخدام سياسة التقييم القائمة على أساس الكم، والتوجه إلى سياسة التقييم على أساس الجودة النوعية المحققة، واعتبارها المعيار الأساسي للتقييم. 7. ت تمية صفة القيادة لدى المديرين.

8. التحسين المستمر للمنتجات والخدمات والابتعاد عن سياسة تحقيق الربح بأية وسيلة فهذه النظرة قصيرة

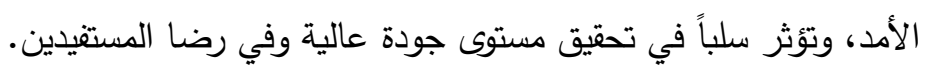

9. إزالة كل العوائق والحواجز التي تمنع العاملين من تحقيق فئق إنجازاتهم والتفاخر بها.

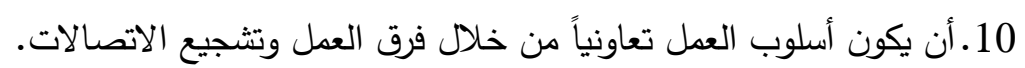

11. التركيز على عملية التطوير الذاتي للعاملين، وإكسابهح معارف ومهارات جديدة.

12.تحفيز العاملين وتوفير عنصر الاستقرار والأمان الوظيفي لهم، وأبعاد شبح الخوف الذي يحد من الإبداع

$$
\text { و الابتكار . }
$$

13.إحداث تغيير جذري في الهيكل التنظيمي للمنظمة، وتحويله من النمط التقليدي إلى نمط حديث مبتكر

$$
\text { يخدم تطبيق النقاط أو المبادئ السابقة. }
$$

14.تجنب ومقاومة الخوف من التغيير والعمل على ترسيخ المبادئ السابقة لاى جميع العاملين، وجعلها

حقيقة وليست مجرد شعارات ينادى بها، وحثهم على تطبيقها والالتزام بها بشكل دائم ومستمر .

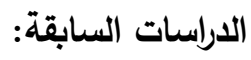

فلسفة ديمنج لإدارة الجودة الثاملة تتلخص في 14 مبدأ وتم تصنيفها إلى ستة عناصر : 1. تحديد أهداف استراتيجية معلنة وواضحة بالمهام والتحول الاداري للجودة وتجنب مقاومة التغيير:

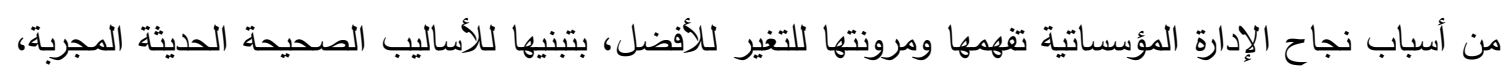
والتخطيط السليم لحاضر ومستقبل المؤسسة كما أثبتت دراسة (بن عيشاوي ا.، 2006) بعنوان "إدارة الجودة الثاملة في المؤسسات الخدماتية " وقد هدفت هذه الدراسة إلى بيان مدى أهمية ودواعي المؤسسات الخدمية بتطبيق هذا المنهج الاداري، وإدماجه في سياساتها واستراتيجياتها، وتتمية الميزة التنافسية لديها؛ لتحقيق الأداء

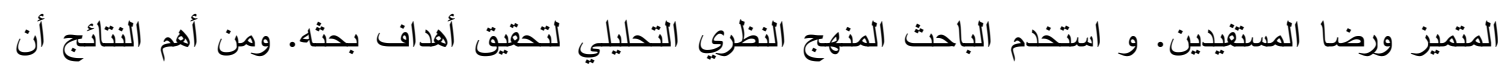

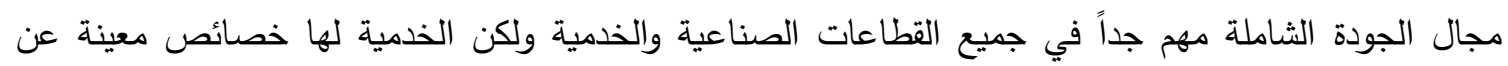
غيرها لذا أوصى الباحثين ببذل مجهودات أكبر في مثل هذه الدراسات وأن الجودة الثاملة شرط أساسي للحصول ولئل على الميزة التتافسية. و وافقه في الاثبات دراسة (الجريفاني، 2008) بعنوان" توجيه الجودة في العمل الخيري خلان خلال بطاقة الأداء المتوازن " والتي تهدف إلى رفع مستويات الجودة والأداء في الجمعيات الخيرية من خلال بطاقة الأداء 
المتوازن من خلال الرؤيا والتخطيط الاستراتيجي لتحقيق أفضل مخرجات للمستفيدين من خلال التطبيق السليم لإدارة الجودة الثاملة. حيث أن من أهم الاستتتاجات أن تحسين الأداء لا يكون إلا من خلال التطبيق الصحيح للتخطيط

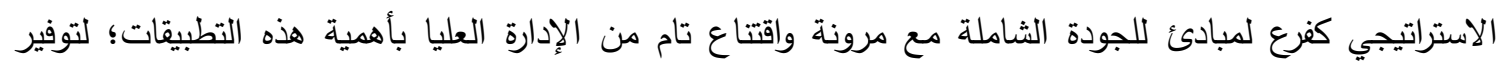
الوقت والجهد والتكلفة.

ومن الناحية التطبيقية كمثال للنجاح التطبيقي دراسة (صالح، 2015) بعنوان" أثر إدارة الجودة الثاملة كاستراتيجية لتطوير الأداء الأكاديمي بجامعة الطائف " والتي تهدف إلى مدى استيعاب أعضاء هيئة التدريس لدفاهيم الجودة وأثر هذا النظام على تطوير الأداء وتكاليفه ومعوقاته. واستخدم الباحث المنهج الوصفي التحليلي

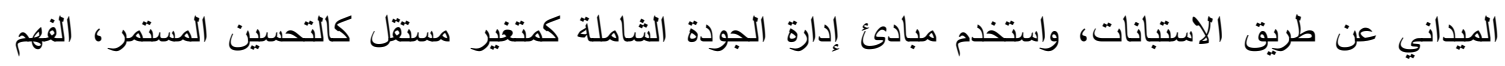
الصحيح لفلسفة الجودة، ضمان توكيد الجودة واستراتيجية التطوير ، ومن الناحية الأخرى استخدم الأداء الأكاديمي كمتغير تابع. ومن أهم نتائج الدراسة أن هناك وعي كبير وحرص من قبل الإدارة بأهمية تتمية وتدريب عضو هيئة الأحئة

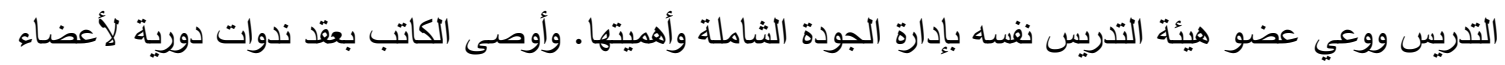
هيئة التدريس من أجل تبادل الخبرات وتحقيق اعتماد أكاديمي أفضل وهذا دليل على قوة التخطيط الاستراتيجي

للإدارة العليا ومرونتها.

ومن الناحية الأخرى لضعف التخطيط الاستراتيجي وعدم وضوح الأهداف فقد أوضحت نتائج دراسة (بن عيشاوي

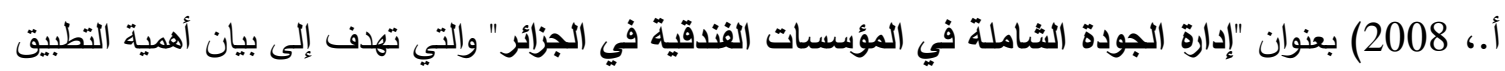

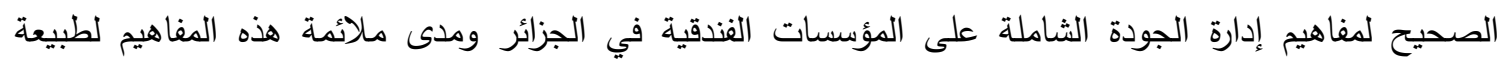
نثاطات الفندقة، مع التعرف على أهم المعوقات وطرق التخلص منها للحصول على النتائج المرجوة، وقياس أثر الثرات إدارة الجودة الثاملة على نثاط الفندق وإكسابه الميزة التنافسية. واستخدم الكاتب المنهج الوصفي التحليلي ودراسة

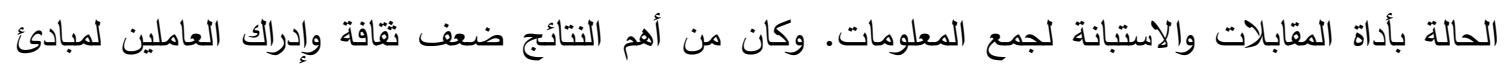

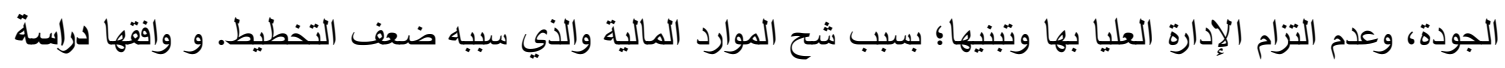

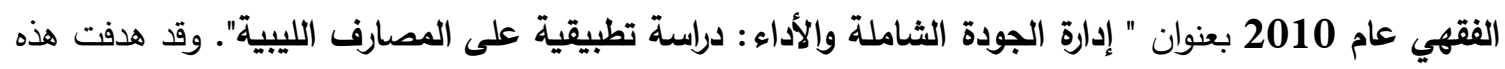

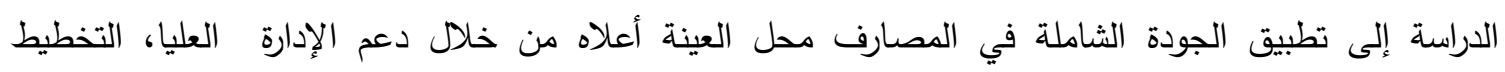

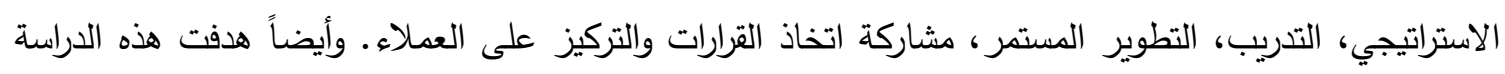

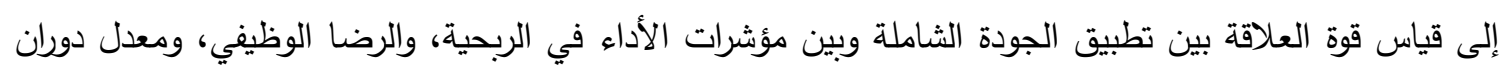

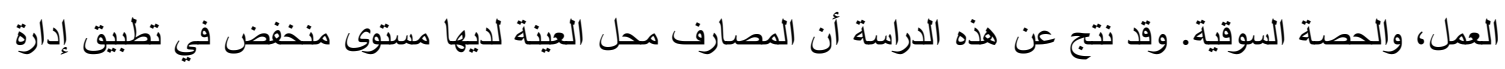
الجودة الثاملة وأن هناك فروق ذات دلالة إحصائية في متوسطات العينة على كل من عناصر الجان الجودة الثاملة وعلى مؤشرات الأداء المؤسسي العام والخاص حيث أن التطبيق الصحيح يقع على عاتق الإدارة العليا بالمقام الأول وتصحيح الانحرافات كسبب لسوء التطبيق ولعدم التحول الصحيح لإدارة الجودة الثاملة و وافقها بالنتائج أيضاً دراسة (عبد ربه، 2014): وهي دراسة بعنوان " التحديات الإدارية التي تواجه الجمعيات الخيرية وسبل مواجهتها دراسة تطبيقية على عينة من الجمعيات الخيرية في سلطنة عمُان". حيث هدفت الدراسة إلى التعرف على أبرز

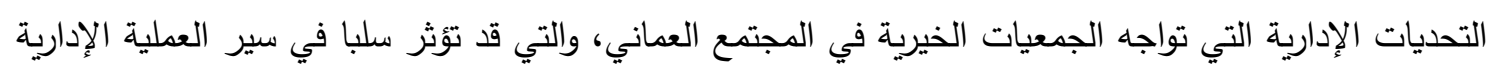
وتعوق قدرتها على القيام بتحقيق أهدافها. بالإضافة إلى البحث عن حلول وإرشادات عامة يمكن من خلالها تقديم التهات مساهمات فعّالة لمواجهة تلك التحديات ـ حيث استخدم الباحث المنهج الوصفي التحليلي (الاستطلاعي)، والاستبيان

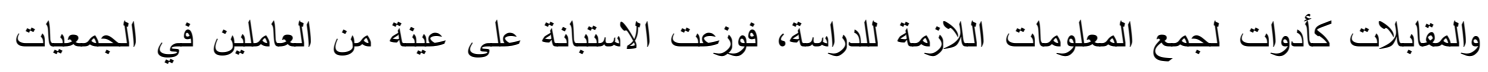
الخيرية بلغ عددها 209 من المبحوثين، بالإضافة إلى مقابلة 15 من المديرين الذين يعملون في ثمان جمعيات 
تعمل في خمس محافظات في سلطنة عمان .وقد توصلت الدراسة للعديد من النتائج أهمها أن الجمعيات الخيرية في سلطنة عمان تواجه تحديات إدارية فيما يتعلق بالتتظيم الإداري، والتوظيف، والتخطيط الإداري والإشراف، والتوجيه،

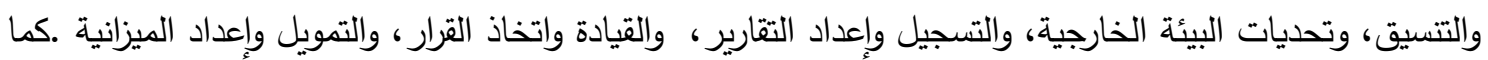

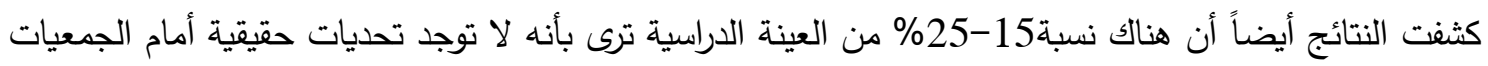

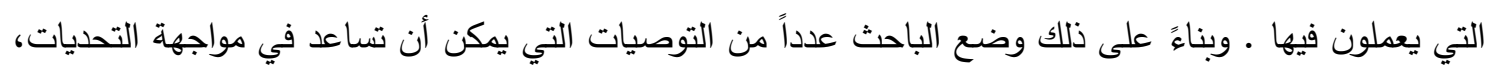
وتتضمن بذل المزيد من الجهود المنظمة على مستوى الجمعيات الخيرية، والمؤسسات التعليمية، ومؤسسات المجتمع. وهذا دليل على وجود خلل كبير في سياسات الإدارة العليا بالتطبيق الصحيح لمبادئ إدارة الجودة

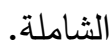

ومن ناحية توافق الدراسات أعلاه مع بحثا هنا فقد اتفق بن عيثاوي وجريفاني وفقهي وصالح في الأهداف والأهمية

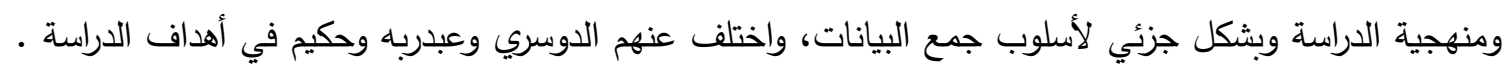

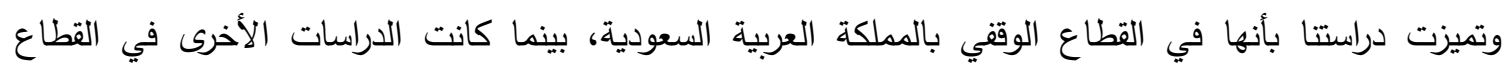
المصارف كدراسة الفقهي، وفي القطاع الفندقي كدراسة بن عيشاوي ودراسة صالح في القطاع التعليمي والآخرين في

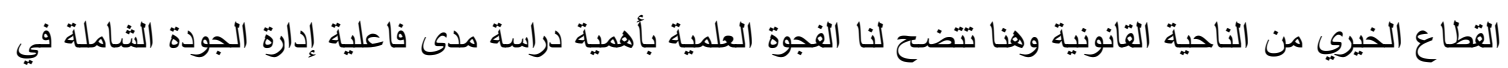
أوقاف جمعيات البر بالمملكة العربية السعودية.

2. الاشتراك في قرارات الجودة و العمل بروح الفريق الواحد:

الاشتراك في قرارات الجودة والعمل بروح الفريق الواحد يعتبر من أساسيات إدارة الجودة الثاملة كما هدفت دراسة

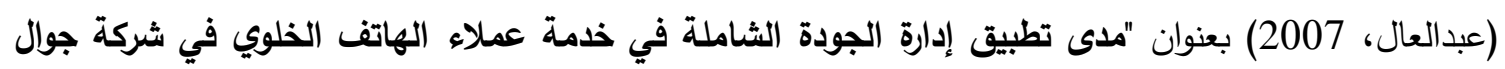
من وجهة نظر العاملين بها في فلسطين" إلى بيان النشاطات الرئيسية لإنجاح تطبيق إدارة الجودة الثاملة في

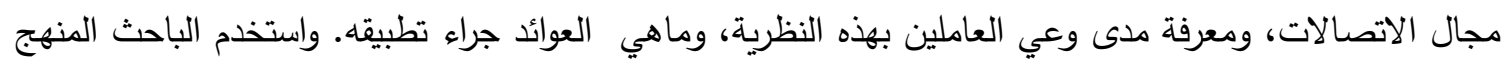
الوصفي التحليلي وباستخدام الاستبانات بحسب المتغيرات المستقلة للعاملين كالعمر ، الجنس والوظيفة. وكانت أبراء أبرز

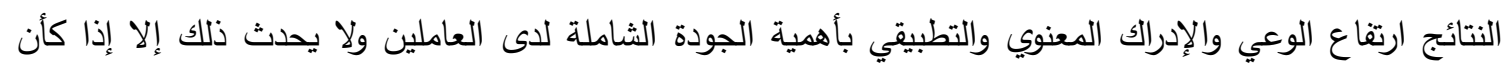
العاملون مشتركين في قرارات الجودة ويعملون بروح الفريق الوحد. وكلاليل للنجاح أثثت دراسة (الجبوري، 2011) بعنوان "أثر تطبيق إدارة الجودة الثاملة على خدمات

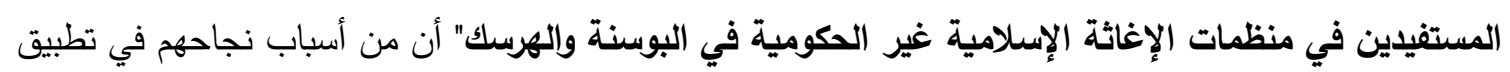
إدارة الجودة الثاملة الاثتراك في اتخاذ قرارات الجودة والعمل بروح الفريق الواحد حيث كأن هدف الكاتب من هذه فئه الدراسة التعرف على أثر إدارة الجودة الثاملة وأهميتها من وجهة نظر العاملين في هيئات الإغاثة الغير حكومية. واستعان الكاتب بالمنهج الوصفي التحليلي والمسحي والتاريخي عن طريق الاستبانات بالمتغيرات المستقلة الثخصية

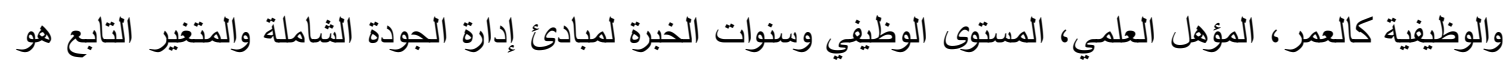

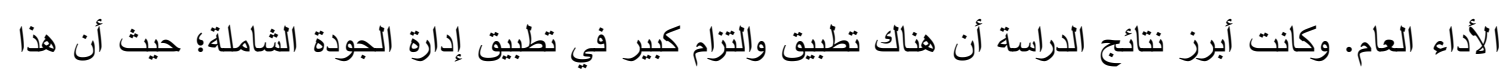
الإجماع بالتطبيق لا يتحقق إلا من خلال الاشتراك في قرارات الجودة والعمل بروح الفريق الواحد. ومن الجاتب الآخر أن عدم اعتماد مشاركة قرارات الجودة مع العاملين والعمل بروح الفريق يؤدي إلى خلى خلل في تطبيق إدارة الجودة الثاملة على المؤسسة كما أثبتت دراسة (بدر، 2009) بعنوان " أثر تطبيق مبادئ الجئ الجودة الثاملة على أداء المؤسسات الأهلية الأجنبية العاملة في قطاع غزة " وهدفت إلى بيان أثر تطبيق إدارة الجودة 
الثاملة على أداء المؤسسات الأهلية وواقع التطبيق ومقاييس الأداء المتبعة. حيث أعتمد الباحث المنهج الوصفي التحليلي وتوزيع الاستبانات على مجتمع البحث بالمستقلات التالية كالتركيز على المستقيدين، التزام الإدارة العليا بالجودة، مشاركة العاملين، السياسات والاستراتيجيات، التحسين والتطوير المستمر والنظم الادارية ومن ناحية

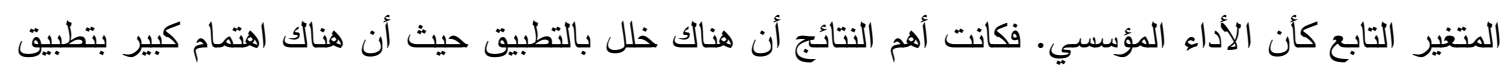

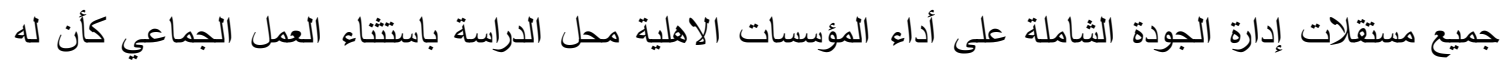
النصيب الاقل في التطبيق.

ومن ناحية توافق الدراسات أعلاه مع بحثنا هنا فقد اتقق Madanat \& Khasawneh و جبوري وبدر في هدف الدراسة والأهمية والمنهجية مع اختلاف جزئي في أداة جمع البيانات واختلفوا في قطاع وجغرافية التطبيق، وهنا تتضح لنا الفجوة العلمية لموضوع دراستنا هنا بأهمية دراسة مدى فاعلية إدارة الجودة الثاملة في أوقاف جمعيات البر بالمملكة العربية السعودية.

3. تبنى الرقابة الوقائية وعدم التقييم على أساس الكم وتجنب القرارات المبنية على التكاليف :

تبنى الرقابة الوقائية وعدم التقييم على أساس الكم وتجنب القرارات المبنية على التكاليف فقط من أساسيات التطبية التبيق

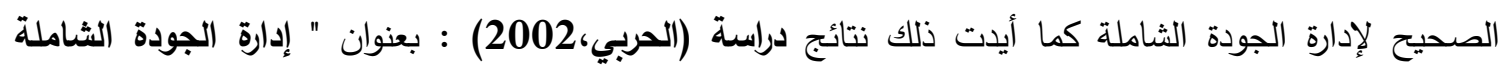
كمدخل لتطوير الجامعات السعودية، دراسة لاتجاهات الأكاديمية السعودية نحو تطبيق مبادئها، ووجهة نظرهم حول مدى إسهام هذا التطبيق في تطوير الجامعة " بالإجماع على أهمية شمولية مقاييس التقييم والتطوير الكائنة

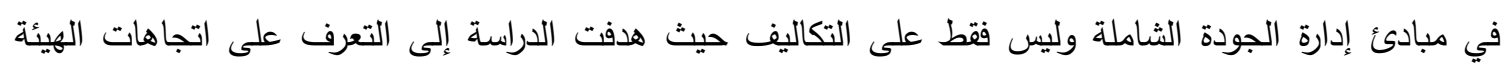
الأكاديمية السعودية نحو مبادئ إدارة الجودة الثاملة، ومدى مساهمة تطبيق هذه المبادئ في تطوير الجامعة من وجهة نظر هذه الهيئة. فانتهجت الباحثة المنهج الوصفي المسحي لتحقيق أهداف دراستها، واستخدمت الاستبيان كأداة لجمع المعلومات والتي وزعتها على مجتمع البحث المتمثل بأعضاء الهيئة التدريسية الذكور والإناث الموزعين

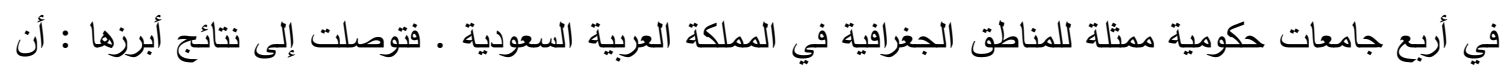
اتجاهات الهيئة الأكاديمية قد مالت باتجاه الموافقة على تطبيق مبادئ إدارة الجودة الشاملة بالجامعات السعودية بدرجة فوق المتوسطة، حيث كانت أبرز المبادئ المتفق على ضرورة تطبيقها، هي التخطيط الاستراتيجي للجودة،

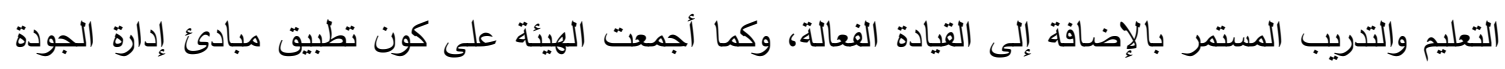

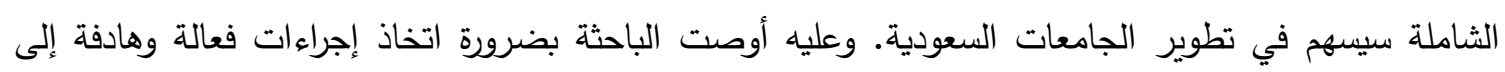
تطبيق مبادئ إدارة الجودة الثاملة في الجامعات السعودية، وإلى ضرورة العمل على تلى توفير التعليم والتخطيط

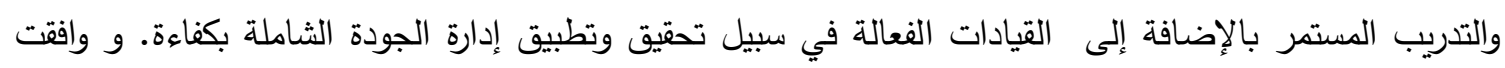

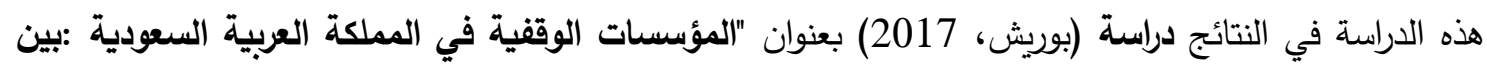

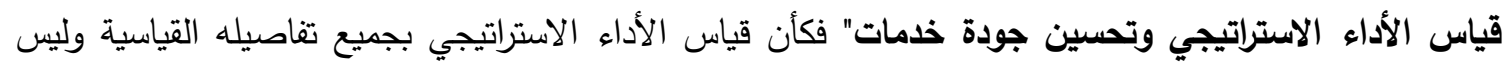

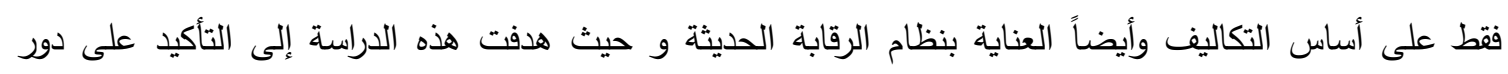
الوقف الفعال في مجتمعاتتا، وأهمية العناية به وبجودته من خلال مقاييس الأداء الاستراتيجية، وتدوير المخرجات.

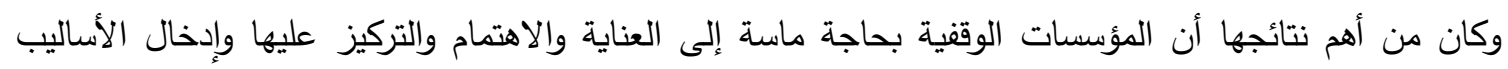
الإدارية الحديثة لتدعيمها، ورقابتها بعناية، ومنعها من أيد العابثين من خلال المنهج الوصفي التحلية التيلي والتاريخي.

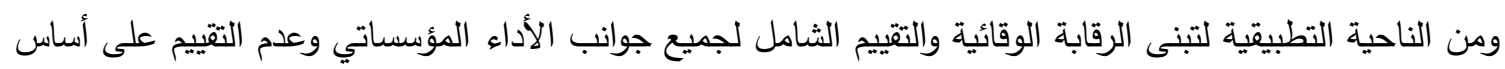
الكم وتجنب القرارات المبنية على التكاليف فقط فقد عنيت دراسة (الثايع، 2013): بعنوان "تقييم مستوى ممارسة التية الجودة الثاملة في الجمعيات الخيرية من وجهة نظر العاملين: دراسة حالة جمعيات الأسرة الخيرية بمدينة بريدة 
والرس". والتي تهدف إلى تثخيص واقع ممارسة مبادئ إدارة الجودة الثاملة في الجمعيات الخيرية لمدينتي بريدة

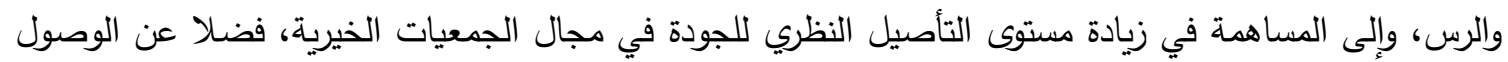

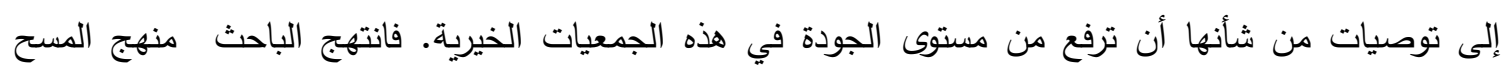

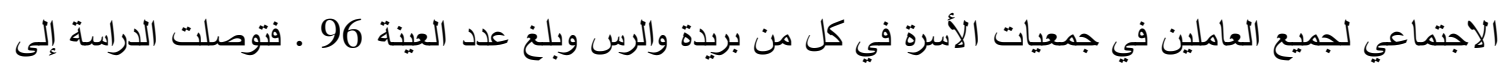

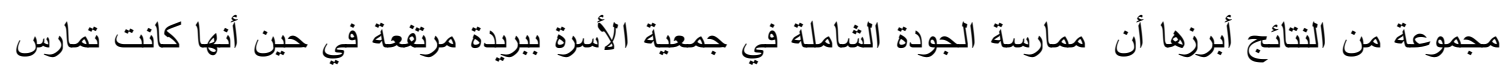
بشكل متوسط في جمعية الرس. كما توصلت هذه الدراسة إلى وجود فروق ذات دلالة إحصائية في ممارسة الجودة

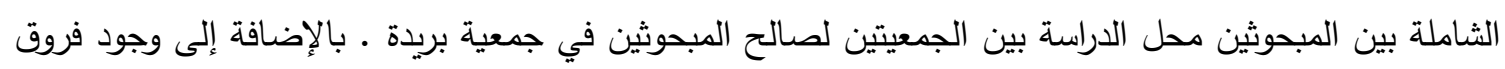

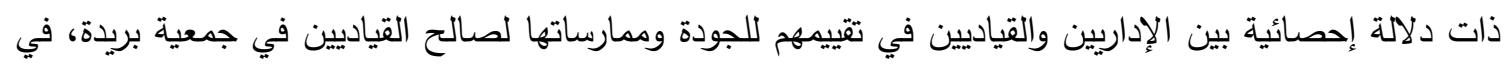
حين لا توجد مثل هذه الفروق فيما بينهم بالنسبة لجمعية الرس. وكانت نتائج المساهمة من مرتفع إلى متوسطة

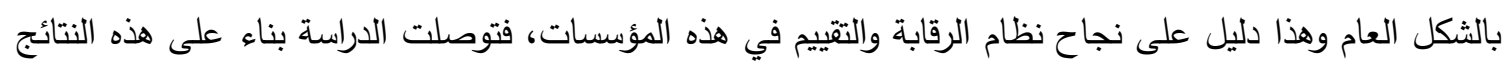
إلى مجموعة من التوصيات من أبرزها أهمية قيام وزارة الثؤون الاجتماعية بإنشاء هيئة وطنية للجودة معنية بتقييم

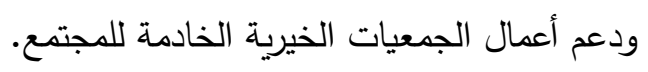
ومن ناحية توافق الدراسات أعلاه مع بحثنا فقد اتفق الحربي وبو ريش والثيات الثايع في هدف الدراسة والأهمية والمنهجية

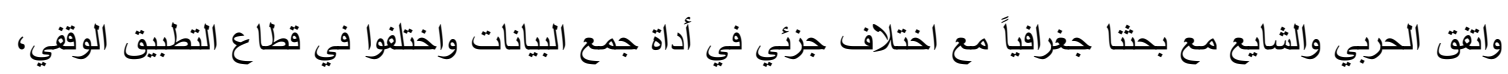

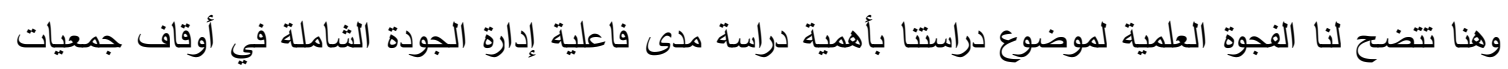
البر بالمملكة العربية السعودية.

4. التدريب المستمر والتركيز على عملية التظوير الذاتي للعاملين وتحفيز العاملين وتعزيز الأمان الوظيفي لديهم وتنمية صفة القيادة لاى المديرين:

التدريب المستمر والتركيز على عملية التطوير الذاتي للعاملين وتحفيز العاملين وتعزيز الأمان الوظيفي لديهم وتتمية

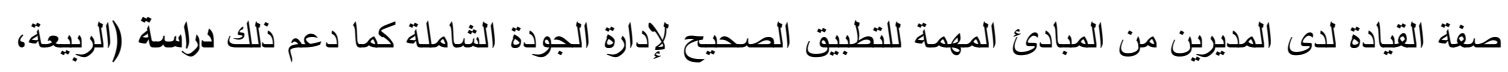

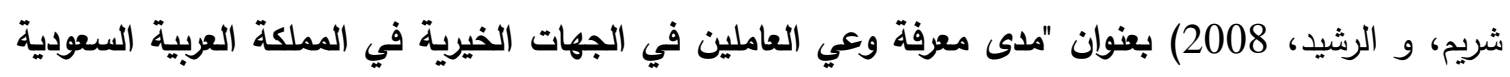
لمفهوم تطبيق الجودة" حيث كأن هدفها معرفة مدى وعي العاملين في الجهات الخيرية في المملكة العربية السعودية لمفهوم تطبيق الجودة. ومن أبرز النتائج التي توصلت إليها الدراسة إدراك غالبية عينة الدراسة بأهمية فئية

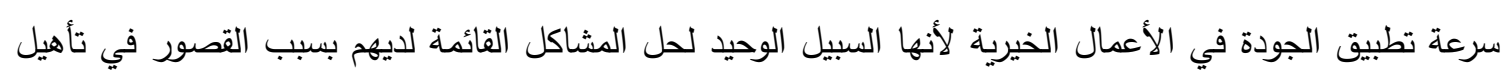
وتدريب العاملين لممارسة الجودة وأبدو الرغبة بالمشاركة والتخطيط وتطبيق الجودة.

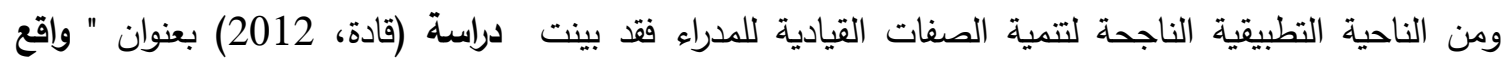

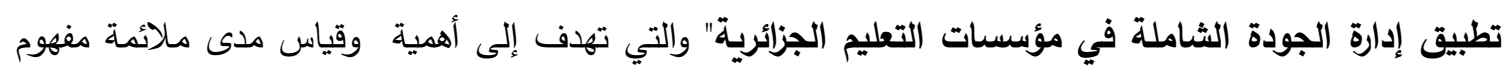

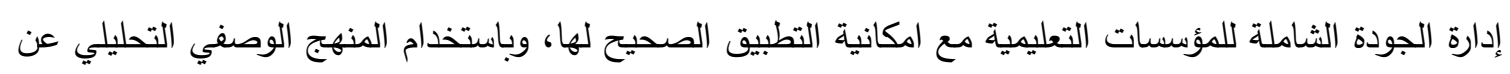
طريق الملاحظات والمقابلات وأيضاً 3 استبانات وزعت على المدراء والأساتذة وطلاب مستوى4 بمتغيرات مستقلة

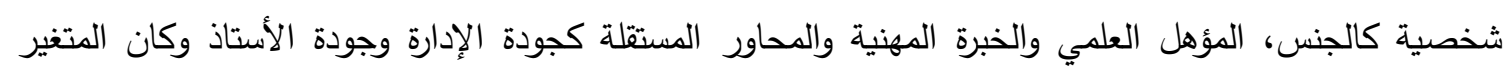

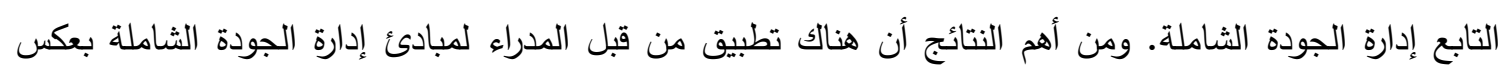

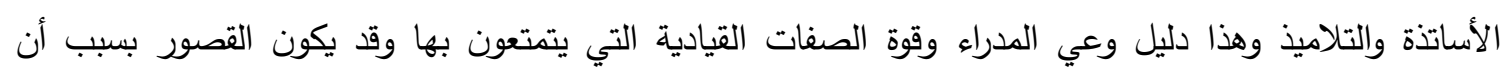
المؤسسة في بداية تطبيق مفهوم إدارة الجودة الثاملة. 
ومن الناحية الأخرى فالنقص في هذا المبدأ يؤدي إلى خلل في تطبيق إدارة الجودة الثاملة كما اثبتت ذلك دراسة (العميره، 2003) بعنوان " علاقة الجودة الثاملة بالأداء الوظيفي في القطاع الصحي" وهدفت إلى معرفة مدى لئى الدعم الذي تقدمه الإدارة العليا من أجل تطبيق ونشر الوعي بأهمية إدارة الجودة الثاملة وما مستوى إدراك العاملين

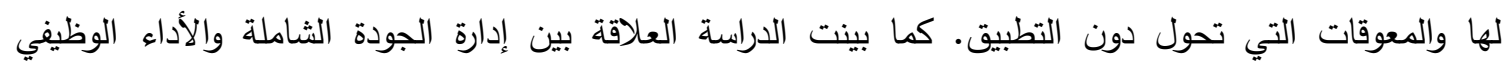
باستخدام المنهج الوصفي التحليل بأداة الاستبانات بمستقلات كالجنس، العمر، الحالة الاجتماعية، الجنسية والمؤهل العلمي. وكان من أبرز النتائج أن هناك تطبيق من جميع الموظفين الإداريين مع خلل في فعالية التطبيق ترجع إلى بلى التئي ضعف برنامج التحفيز. وقد أوصى الكاتب بمراعات جوانب الخلل. و وافقه (الخراشي، 2008) بعنوان بحثه

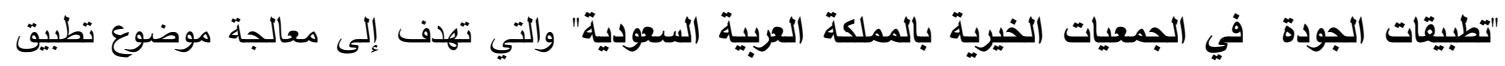

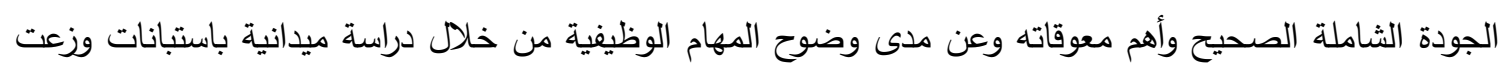

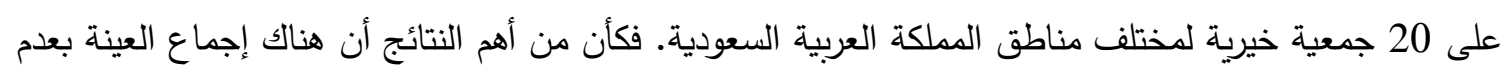

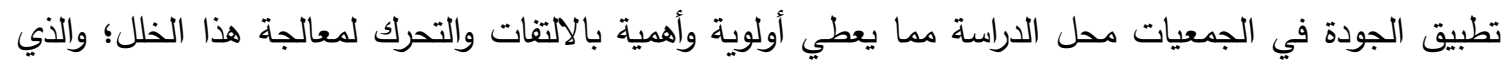

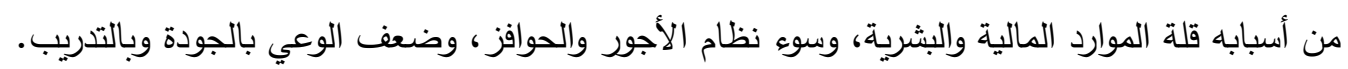

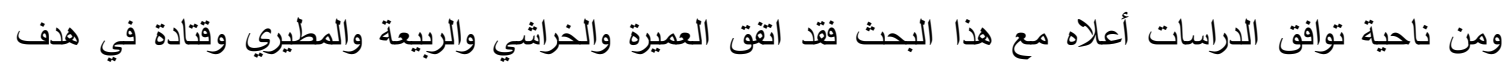

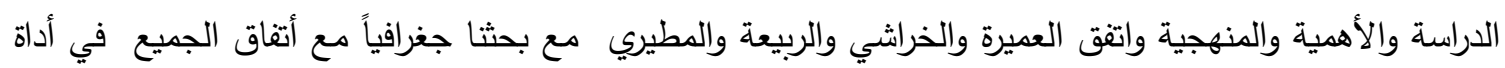

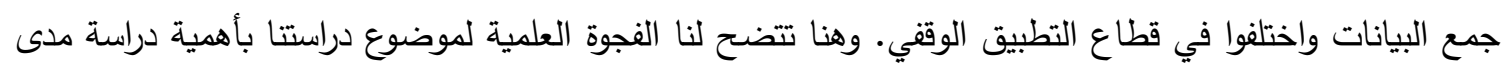
فاعلية إدارة الجودة الثاملة أوقاف جمعيات البر بالمملكة العربية السعودية. 5. التحسين المستمر للمنتجات والخدمات:

التحسين المستمر للمنتجات والخدمات من أهم عوامل الاستدامة ومقاومة المنافسين وهي من أحد أعمدة مبادئ إدارة

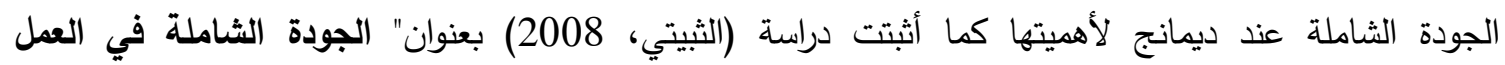
الخيري.. المستفيد أولاً" والتي تهدف إلى التركيز على زيادة الوعي بأهمية التركيز على المستفيد أولأ من الخدمات الخهات

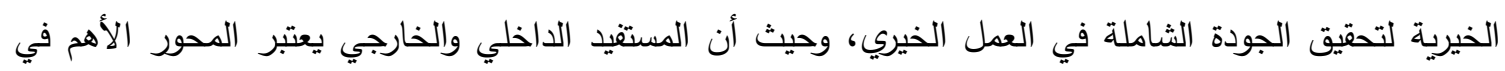
إدارة الجودة الثاملة ليقدم له أفضل الخدمات التي يطمح لها، وشملت الدراسة تفصيل لهؤلاء المستفيدين

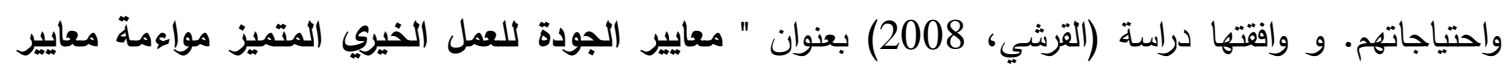

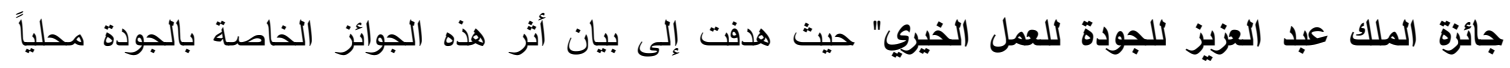

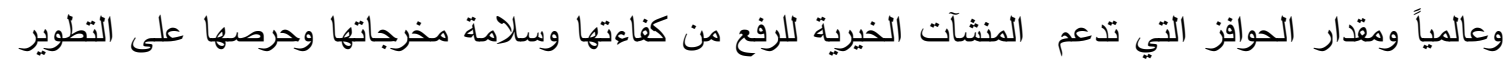
المستمر وقدرتها على المنافسة. ومن أهم نتائجها إيضاح المعايير الخاصة بهذه الجائزة وكيفية تطبيقها لمساعدة الجمعيات الخيرية على التطبيق الصحيح للجودة للوصول إلى المراتب المتقدمة في الجائزة محلياً وعالمياً. ويعتبر هذا من المحفزات على التطبيق الصحيح للجودة للتحسين المستمر في المنتجات والخدمات ونابع أيضاً من اهتمام

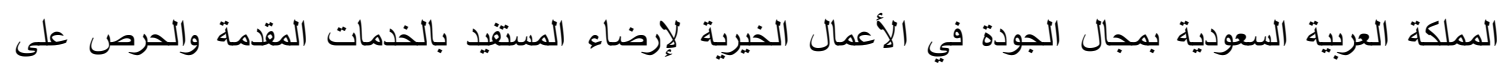
التحسين والتطوير المستمرين. و واففتها دراسة (العتيبي، 2010) بعنوان "مدى الالتزام بتطبيق معايير إدارة الجودة

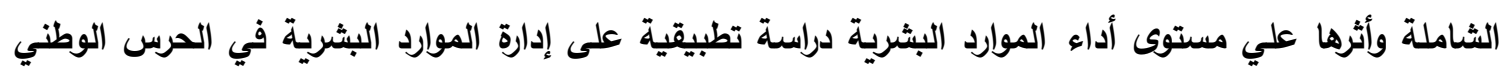

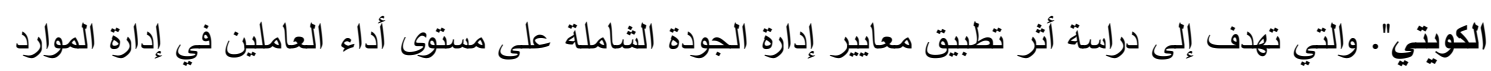
البشرية بالحرس الوطني الكويتي، بالإضافة إلى التعرف على الاختلاف في تصور عينة الدراسة البالغة 75 رتبة عن طريق المنهج الوصفي التحليلي لتطبيق معايير إدارة الجودة الثاملة باختلاف العمر والرتبة. وكانت النتائج 
متراوحة من مرتفع إلى متوسط. وقد أوصى الباحث بأهمية تطبيق الجودة الثاملة والاستفادة بشكل أساسي من اقتراحات المستفيدين عن أداء الموظفين وكل ذلك من بهدف التطوير والتحسين المستمر للخدمات المقدمة. و وافقته دراسة (زديرة، 2017) بعنوان " دور إدارة الجودة الثاملة في تحسين جودة خدمات المؤسسات الاستشفائية"

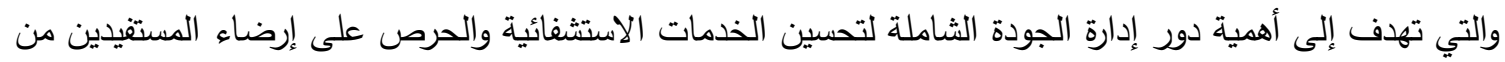
خلال تبني هذه النظرية وزرعها في نفوس الموظفين ببيان أهميتها وبالتدريب والتحفيز • وقد استخدم الباحث المنهج الوصفي التحليلي عن طريق الاستبانات بالمتغيرات المستقلة التالية كالاعتمادية، الاستجابة، الضمان، الملموسية

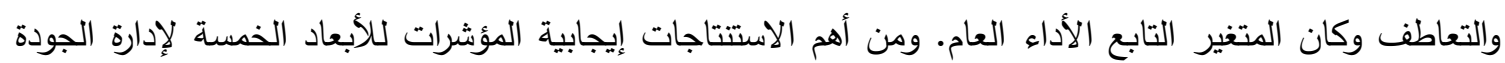
The Impact of The " بعنوان الثاملة في التطبيق بثكل كبير • وأيدهم دراسة (Lisda \& Firdaus, 2017) Application of Total Quality Management on The Quality of Service and The Performance of Islamic Banks " والتي تهدف إلى دراسة آثار تطبيق إدارة الجودة الثاملة على جودة الخدمة وأداء البنوك الإسلامية من خلال المنهج الوصفي التحليلي وأداة الاستبانات. ومن أهم نتائج هذه الدراسة

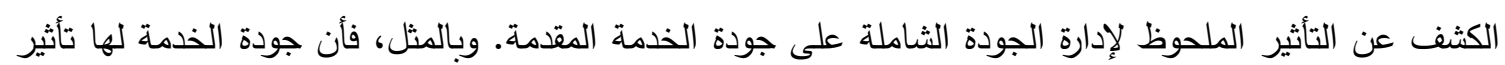

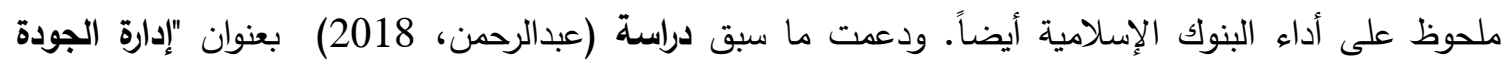

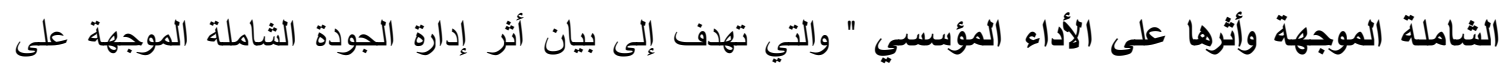

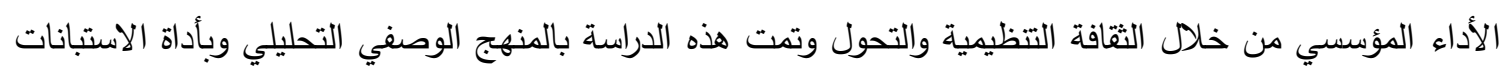

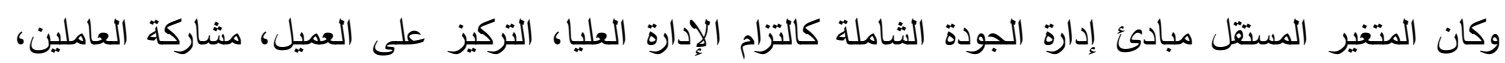

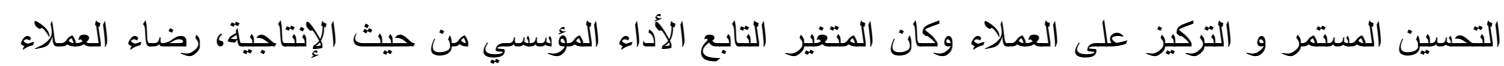

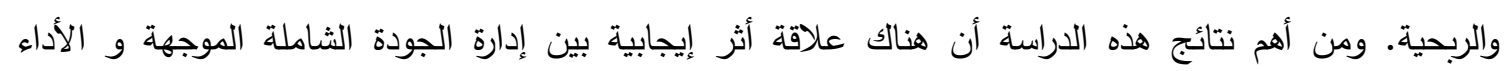
المؤسسي، فكلما كأن الأداء المؤسسي في أعلى مستوياته انعكس ذلك على جودة التهائ الخدمة المقدمة ومن ثم إرضاء المستفيد.

ومن الناحية التطبيقية هناك دراسات أثبتت نجاح هذا المبدئ بنتائج إيجابية متوسطة وفوق المتوسطة

كدراسة (زقزوق، 2008) بعنوان "تطبيق مبادئ إدارة الجودة الثاملة لتحسين أداء كلية خدمة المجتمع والتعليم

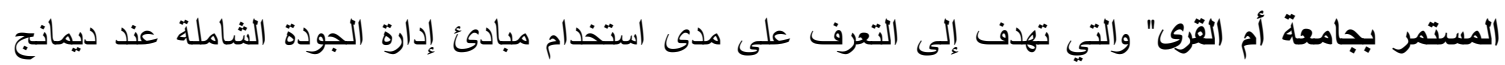
والتعرف على أهمية تطبيقها. حيث أستتتج الكاتب أن مدى الالتزام ودرجة الاهتمام بتطبيق مبادئ إدارة الجودة

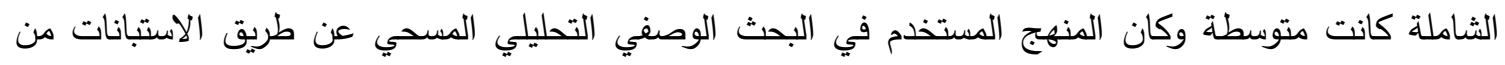

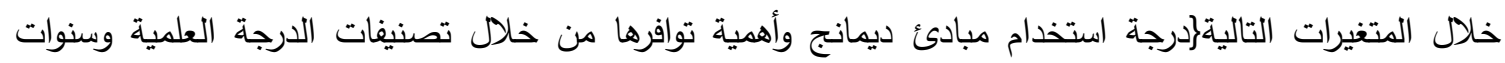
الخبرة وبلد التخرج). و وافقه أيضاً (لثرف، 2011)بدراسته بعنوان "معايير إدارة الجودة الثاملة كداعم لجودة

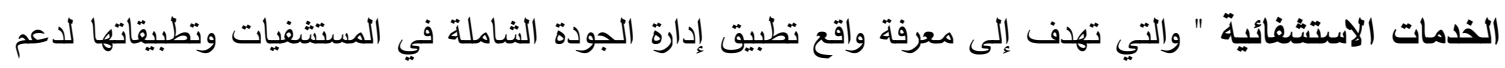

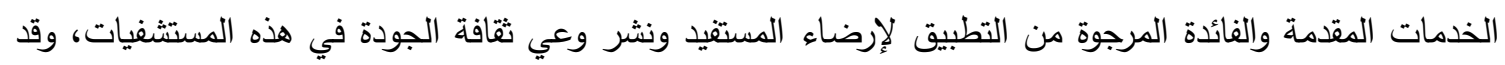

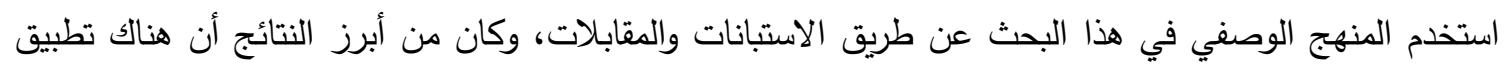

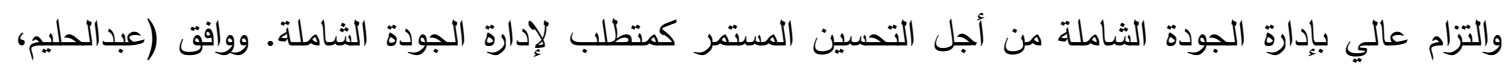
2012) من قبله بدراسة "تحسين أداء المؤسسة في ظل إدارة الجودة الثاملة " والتي تهدف إلى محاولة فهم الحلول المناسبة للرفع من أداء المؤسسات عن طريق إدارة الجودة الثاملة وفهم التطبيق الصحيح والفعال لها بالفهم العميق لأسرار تفوق اليابانيين في التطبيق الصحيح والفعال، ومعرفة أهم الصعوبات والعراقيل التي تحول دون التطبيق الأمثل. والمنهج المتبع كان الوصفي التحليلي عن طريق الاستبانات و بالمتغيرات المستقلة التالية كالمحور 
المالي، محور الزبائن، محور العمليات، محور التعلم و التطوير والمتغير التابع كأن الأداء المؤسسي عن طريق

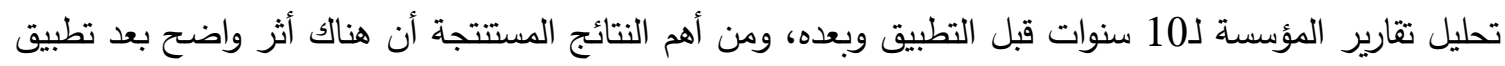
إدارة الجودة الثاملة من حيث ازدياد الاهتمام بالمجتمع والمستفيدين بثكل كبير وملحوظ. و وافقهم دراسة (سيد و ولهن التميمي، 2013) بعنوان " تطبيق إدارة الجودة الثاملة لتحسين أداء العاملين " والتي تهدف إلى بيان أثر تطبيق دائيق إدارة الجودة الثاملة لتحسين أداء العاملين في المستثفيات الجزائرية وأهميته الملحة، واستخدم الباحثان المنهج الوصفي التحليلي اعتمادا على الاستبانات كأداة لجمع البيانات. وكان المتغير المستقل لدراستهما مبادئ إدارة الجودة

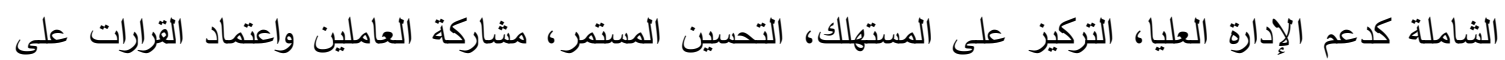
البيانات بينما كأن المتغير المستقل أداء العاملين كمستوى الرضا الوظيفي، مستوى الكفاءة التشغيلية ومستوى خدمة التهاء الإدارة. ومن أهم نتائج الدراسة أن هناك استعداد وتوجه تام و اقتتاع من قبل العاملين في المستثفيات محل الدراسة من أجل تحسين أداءهم. مع توصية الباحثان بضرورة الإسراع في التطبيق وتسهيل كل الاجراءات والعقبات التي وني تحول دون ذلك، وهذه الأبحاث الآنفة الذكر داعم كبير لعملية التحسين المستمر الذي يقود بطريقه إلى التطبيق الصحيح لففهوم إدارة الجودة الثاملة. ومن ناحية توافق الدراسات أعلاه مع بحثا هنا فقد اتقق المصري، الثبيتي، القرشي، العتبيبي، لشرف، عبد الحليم،

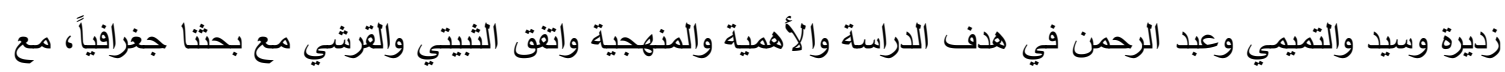

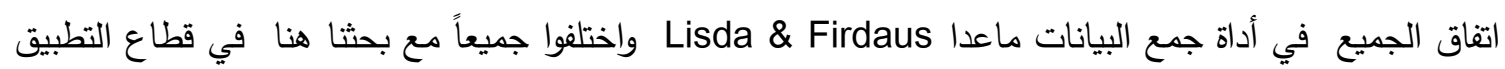
الوقفي. وهنا تتضح لنا الفجوة العلمية لموضوع دراستتا بأهمية دراسة مدى فاعلية إدارة الجودة الثاملة أوقاف جمعيات البر بالمملكة العربية السعودية.

6. إزالة كل العوائق والحواجز التي تمنع العاملين من تحقيق إنجازاتهم والتفاخر بها:

إزالة كل العوائق والحواجز التي تمنع العاملين من تحقيق إنجازاتهم والتفاخر بها شرط أساسي ومبدأ مهم للتطبيق الصحيح لإدارة الجودة الثاملة والاخلال به يؤدي إلى خلل في التطبيق الصحيح وظهور المشاكل، كما أثبتت ذلك التك دراسة (حبشي، 2007) بعنوان " إدارة الجودة الثاملة مع دراسة تطبيقية في وحدة فرمال لإنتاج الأدوية قسنطينة" والتي تهدف إلى التعرف على مدى إدراك العاملين بطبيعة وأهمية إدارة الجودة الثاملة وتقييم الأداء العام ومدى توافقه مع مبادئ إدارة الجودة الثاملة من خلا المنهج الوصفي التحليلي عن طريق الملاحظات والمقات المابلات

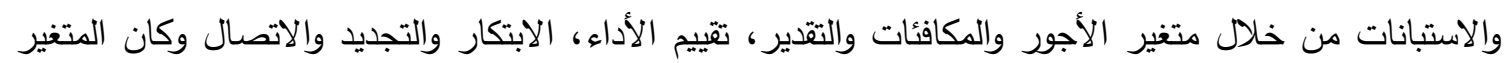

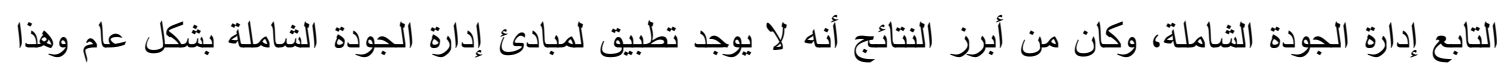
دليل قوي على تدني مستوى نظام إدارة الموارد البشرية مما سبب عوائق وحواجز تمنع العاملين من تحقيق إنجازاتهم. و وافقه (العضاضي، 2012) : بدراسة بعنوان "معوقات تطبيق إدارة الجودة الثاملة في مؤسسات التعليم العالي: دراسة ميدانية". و ولقد هدفت هذه الدراسة إلى معرفة معوقات تطبيق الجودة في مؤسسات التعليم العالي • وانتهج

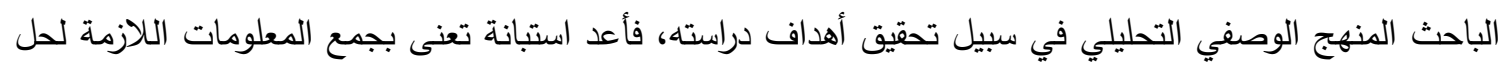

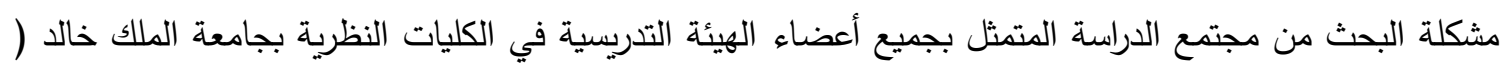

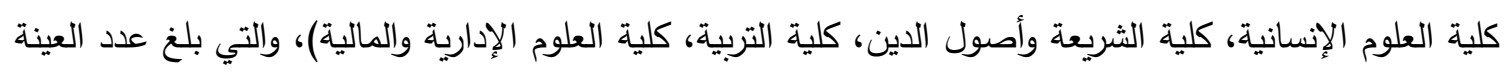

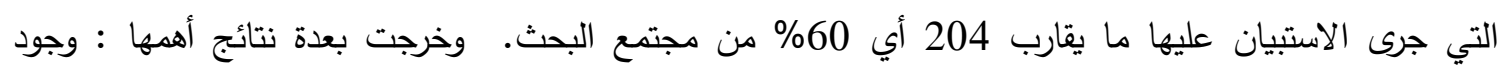
اختلاف في درجة الموافقة على مبادئ إدارة الجودة بين أفراد عينة البحث، إلا هناك إجماع العئ العينة على أن أبرز 
المعوقات التي تواجه تطبيق مبادئ إدارة الجودة الثاملة في مؤسسات التعليم العالي هي : زيادة العبء التدريسي،

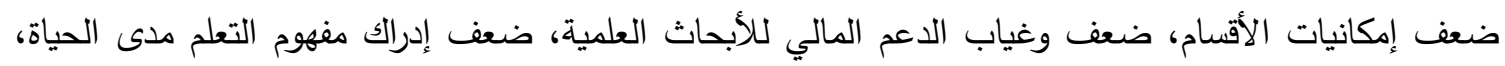

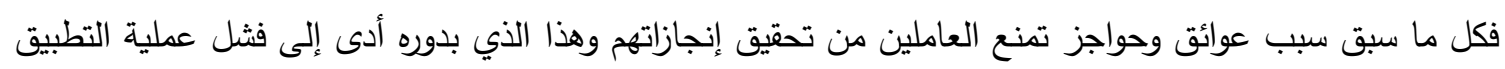
الصحيح لإدارة الجودة الثاملة.

ومن ناحية توافق الدراسات أعلاه مع بحثنا هنا فقد اتفق حبشي وعضاضي في هدف الدراسة والأهمية والمنهجية

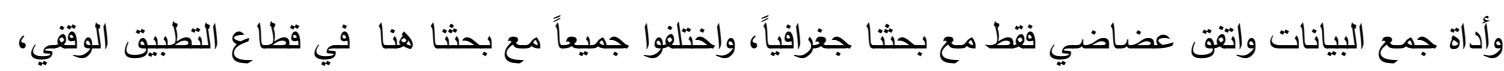

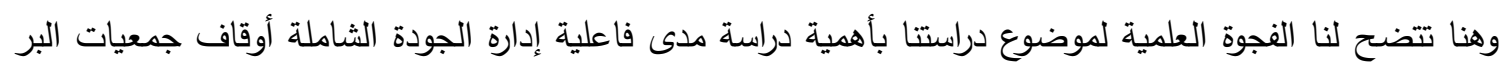
بالمملكة العربية السعودية. خلاصة الاراسات السابقة:

وفي ختام الدراسات تأتي هذه الدراسة مكملة للدراسات السابقة في المجالات المختلفة من خلال دراسة قياس فاعلية إدارة الجودة الثاملة على الأوقاف في المملكة العربية السعودية والتي ستكون بإذن الله إضافة مهمة للمكتبة البحثية وللباحثين...

\section{النتائج والتوصيات والخاتمة:}

يهدف هذا البحث إلى قياس فاعلية تطبيق إدارة الجودة الثاملة على أوقاف جمعيات البر في المملكة العربية السعودية بالثكل الصحيح من خلال ال 14 مبدئ من مبادئ إدارة الجودة الثاملة لديمنج حيث أن هذه المبلى المبادئ الطريق الخصب والمعبد والمجرب للنجاح والتقدم والتطور والتتمية المستدامة من خلال وضوح التئ التؤيا المؤسسية

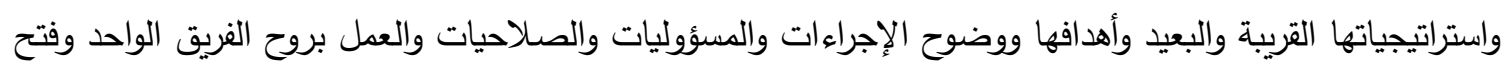

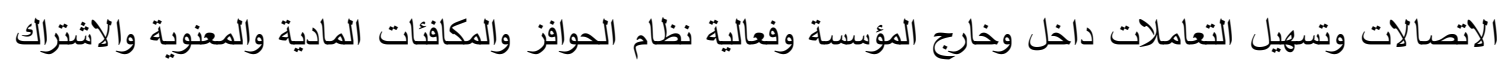

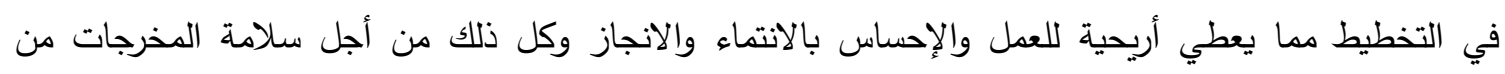

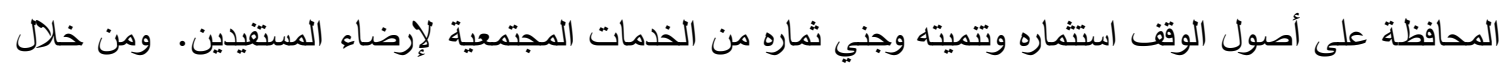

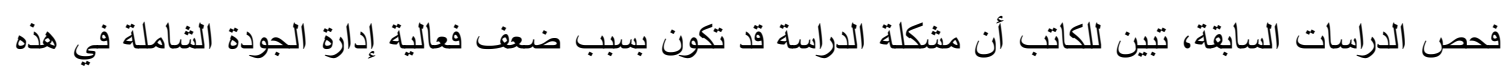

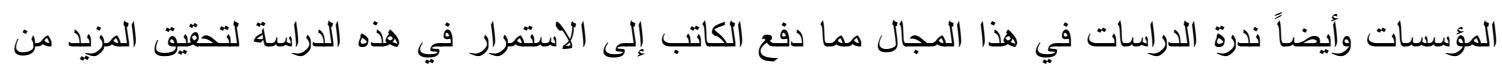
الأهداف والإجابة على أسئلة البحث. مع التوصية بالمزيد من الاهتمام بمجال الجودة الثاملة وتطبيقه بالثكل

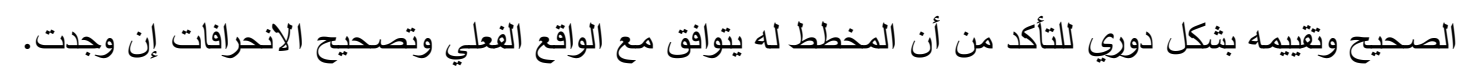

المراجع

الثبيتي، زبن بن عيضة. (2008):"الجودة الثاملة في العمل الخيري.. المستفيد أولاً"، الجودة والعمل الخيري، اللقاء السنوي التاسع السنوي بالمنطقة الشرقية، السعودية.

الثميري، عبدالسلام.(2016):" 81 \% من مشكلات الأوقاف اجتهادات محاكم وسوء إدارة"، أخبار وتقارير، الصحيفة الاقتصادية، الرياض، السعودية. 
الحربي، حياة محمد سعيد. (2002): "إدارة الجودة الثاملة كمدخل لتطوير الجامعات السعودية ، دراسة لاتجاهات الأكاديمية السعودية نحو تطبيق مبادئها ، ووجهة نظرهم حول مدى إسهام هذا التطبيق في تطوير الجامعة" ، رسالة دكتوراة غير منشورة ، جامعة أم القرى ، مكة المكرمة .

الجبوري، جمال مجمود.(2011): "أثر تطبيق إدارة الجودة الثاملة على خدمات المستفيدين في منظمات الإغاثة الاسلامية غير الحكومية"، جامعة أم درمان الإسلامية، السودان.

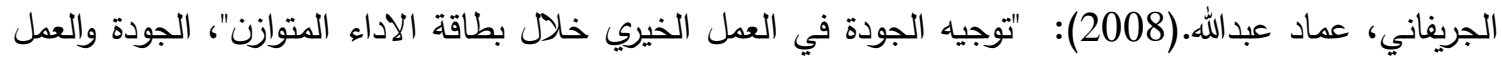
الخيري، اللقاء السنوي التاسع للجهات الخيرية بالمنطقة الشرقية، السعودية. الخراشي، عبدالله بن عثمان.(2008): "تطبيقات ادارة الجوذة في الجمعيات الخيرية." الجودة والعمل الخيري، اللقاء السنوي التاسع للجهات الخيرية بالمنطقة الثرقية، السعودية. الربيعة، عبدالله محمد ؛ شريم، حسن محمد ؛ و الرشيد، محمد ناصر .(2008) : "مدى معرفة وعي العاملين في الجهات الخيرية في المملكة العربية السعودية لمفهوم تطبيق الجودة"، الجودة والعمل الخيري، اللقاء السنوي التاسع للجهات الخيرية بالمنطقة الشرقية، السعودية.

الثايع، محمد عبدله ـ (2013): " تقييم مستوى ممارسة الجودة الثاملة في الجمعيات الخيرية من وجهة نظر العاملين : دراسة مطبقة على جمعيات الأسرة بمدينتي بريدة و الرس" مجلة العلوم الإنسانية ، جامعة

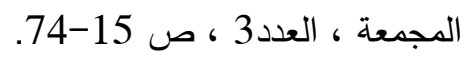

الصالح، محمد بن احمد.(2001) : "الوقف في الثريعة الإسلامية وأثثه في تتمية المجتمع"، المجلد الأول، مكتبة الملك فهد الوطنية، الرياض، السعودية. الصلاحات، سامي.(2013) : "الجودة الوقفية،دراسة في معايير الإدارة والتتمية"، مجلة الشريعة والدراسات

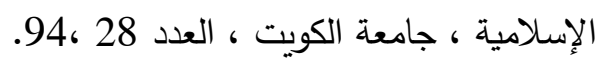
العتيبي، فالح شجاع.(2010) :"مدى الالتزام بتطبيق معايير ادارة الجودة الثاملة واثرها علي مستوى اداء الموارد

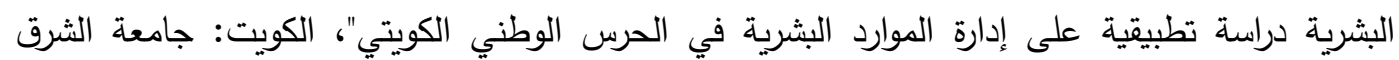
الاوسط.

العضاضي، سعيد بن علي. (2012): "معوقات تطبيق إدارة الجودة الثاملة في مؤسسات التعليم العالي دراسة

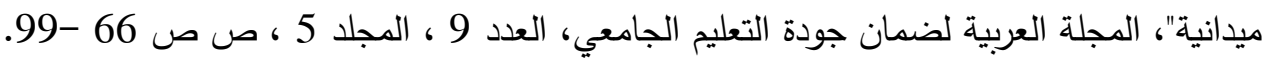
العرابي، حمزة ، و نوارة إيمان.(2016) :"دور إدارة الجودة الثاملة في تعزيز حوكمة الثركات"، مجلة الأبحاث القتصادية العدد 2، 14 6.

العميره، محمد بن عبدالعزيز .(2003) :"علاقة الجودة الثاملة بالأداء الوظيفي في القطاع الصحي"، مركز الامير سلطان لمعالجة وجراحة القلب للقوات المسلحة بمدينة الرياض، السعودية.

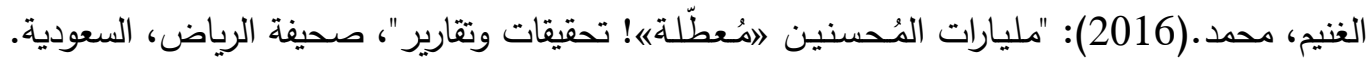
القرشي، حسين بن رده.(2008) : "معايير الجودة للعمل الخيري المتميز مواءمة معايير جائزة الملك عبد العزيز للجودة للعمل الخيري"، الجودة والعمل الخيري، اللقاء السنوي التاسع للجهات الخيرية بالمنطقة الثرقية، السعودية. 


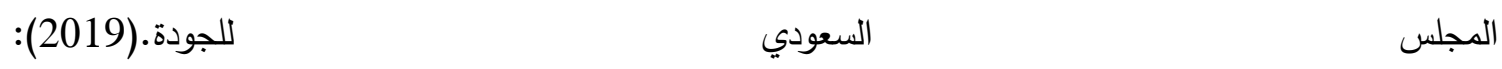

\&id=114\&view=article\&https://www.sqc.org.sa/index.php?option=com_content (تاريخ الوصول 4 8, 2020). lang=sa\&Itemid=83

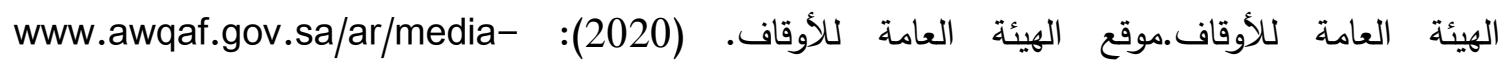

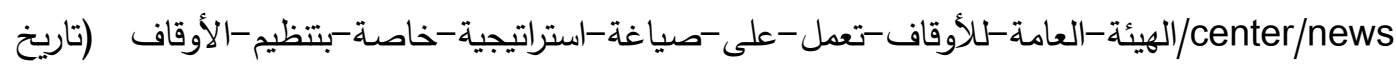
الوصول 12 10, 2020).

بدر، رشاد محمود.(2009): "أثر تطبيق مبادئ الجودة الثاملة على أداء المؤسسات الأهلية الأجنبية العاملة في

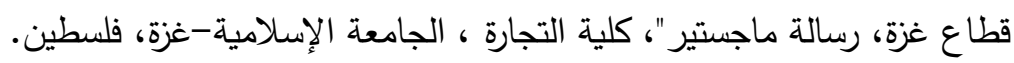

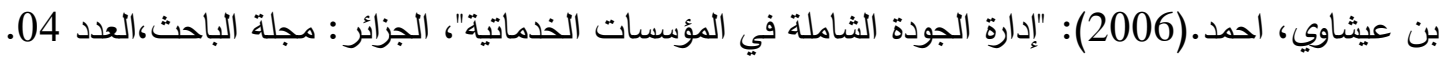
بن عيثاوي، أحمد.(2008): "إدارة الجودة الثاملة في المؤسسات الفندقية في الجزائر"، جامعة الجزائر ، الجزائر . بوريش، هشام.(2017) :"المؤسسات الوقفية: بين قياس الأداء الاستراتيجي وتحسين جودة خدماتها"، جامعة الامير عبدالقادر للعلوم الاسلامية، الأردن.

حبثي، فتحية.(2007) : "إدارة الجودة الثاملة مع دراسة تطبيقية في وحدة فرمال لإنتاج الأدوية قسنطينة"،جامعة منتوري قسنطينة، الجزائر .

حسن، نوبي محمد.(2011): "الوقف والعمران الإسلامي" المجلد الاول،جامعة الملك سعود، الرياض، السعودية.

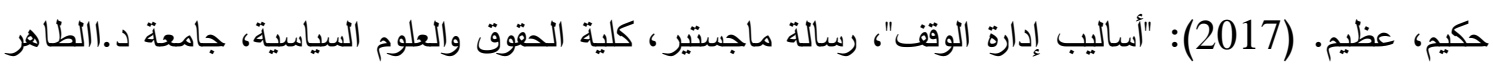
مولاي سعيدة، الجزائر.

زديرة، شرف الدين.(2017) : "دور إدارة الجودة الثاملة في تحسين جودة خدمات المؤسسات الاستشفائية"، جامعة محمد خيضر بسكرة، الجزائر.

زقزوق، خالد جميل.(2008): "تطبيق مبادئ إدارة الجودة الثاملة لتحسين أداء كلية خدمة المجتمع والتعليم المستمر بجامعة أم القرى"،جامعة ام القرى، لمكة المكرمة.

سيد، أحمد؛ حاج، عيسى ؛ و التميمي، وفاء صبحي صالح.(2013) : "تطبيق إدارة الجودة الثاملة لتحسين أداء العاملين"، جامعة البليدة، الجزائر .

شيهاني، سهام.(2010): مداخلة بعنوان : "إمكانية تطبيق نظام إدارة الجودة الثاملة في التعليم العالي-الملتقى جلئرة

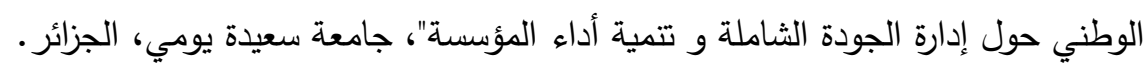

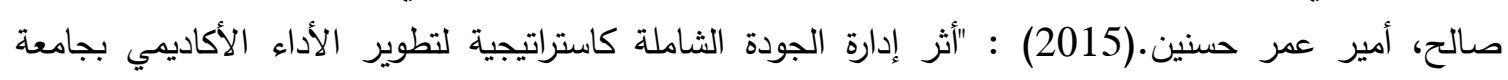
الطائف." مجلة الشرق للدراسات والبحوث العلمية، 9.

عبد الله، طارق.(2012) : "آفاق مستقبل الوقف في تونس"، ندوة الوقف في تونس:الواقع وبناء المستقبل، 28-29 دون فبراير • تونس.

عبدالحليم، مزغيش.(2012) :"تحسين أداء المؤسسة في ظل إدارة الجودة الثاملة"، جامعة الجزائر ، الجزائر .

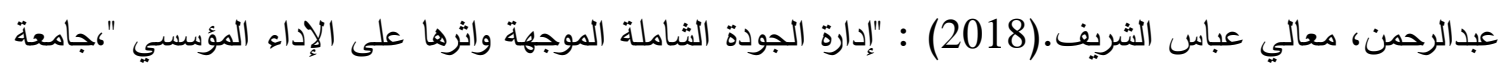
السودان للعلوم والتكنولوجيا، السودان.

عبدالعال، حازم ياسر •(2007): "مدى تطبيق إدارة الجودة الثاملة في خدمة عملاء الهاتف الخلوي في شركة جوال من وجهة نظر العاملين بها في فلسطين"، الجامعة الاسلامية، المدينة المنورة. 
عبدالعظيم، حمدي.(2009): "النتائج المترتبة على تهميش الوقف الإسلامي"، المؤتمر الثالث للأوقاف، الوقف الإسلامي" اقتصاد، وإدارة، وبناء حضارة". الجامعة الإسلامية، السعودية.

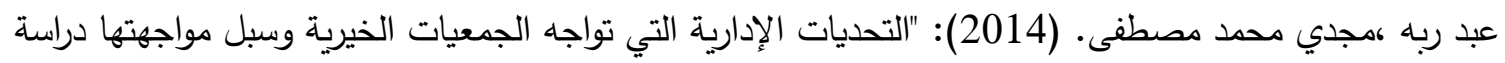
تطبيقية على عينة من الجمعيات الخيرية في سلطنة عمُان"، مجلة الآداب والعلوم الاجتماعية ، مجلد 2 ، 2 ، العدد 5، جامعة السلطان قابوس ، سلطنة عمان.

عقيلي، عمر وصفي.(2001) : "المنهجية المتكاملة لإدارة الجودة الثاملة (وجهة نظر)"، دار وائل للنشر، عمان، الاردن.

قادة، يزيد·(2012): " واقع تطبيق ادارة الجودة الثاملة في مؤسسات التعليم الجزائرية"، جامعة ابي بكر بلقيد، الجزائر

لشرف، نسيمة.(2011): "معايير إدارة الجودة الثاملة كداعم لجودة الخدمات الإستثفائية"، جامعة قاصدي مرباح،

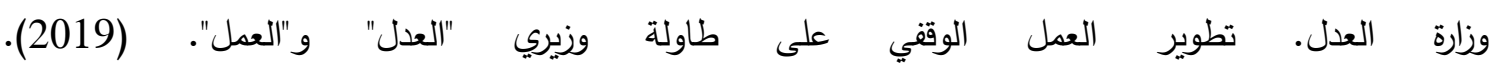
www.moj.gov.sa/ar/MediaCenter/News/Pages/NewsDetails.aspx?itemld=941

وكالة الأنباء السعودية. غرفة الرياض تلقي الضوك على تحديات قطاع الأوقاف. (2019). newsid=1984356\&www.spa.gov.sa/viewfullstory.php?lang=ar

.$(2020$

\section{References}

Abd Rabh, Majdi Muhammad Mustafa. (2014). Administrative challenges facing charitable societies and ways to confront them an applied study on a sample of charitable societies in Oman, Journal of Arts and Social Sciences, 2(5). (in Arabic)

Abdel Aal, Hazem Yasser. (2007). The extent of implementation of total quality management in serving cell phone customers in a Jawwal Company from the viewpoint of its employees in Palestine, The Islamic University, Madinah. (in Arabic)

Abdel Azim, Hamdi. (2009). The consequences of marginalizing the Islamic endowment, The Third Conference of Endowments, The Islamic Endowment: "Economy, Administration, and Building of Civilization". The Islamic University, Saudi Arabia. (in Arabic)

Abdel Halim, Mezgish. (2012). Improving institution performance under total quality management, University of Algiers, Algeria. (in Arabic)

Abdul Rahman, His Excellency Abbas Al-Sharif. (2018). Total quality oriented management and its impact on institutional performance, Sudan University of Science and Technology, Sudan. (in Arabic)

Abdullah, Tariq. (2012), Prospects for the future of the Waqf in Tunisia, The Endowment Symposium in Tunisia: Reality and Building the Future, February 2829. Tunisia. (in Arabic) 
Adadhi, Saeed bin Ali. (2012). Obstacles to the application of total quality management in Higher Education Institutions, a Field Study, The Arab Journal for Quality Assurance of University Education, 5(9), 66-99. (in Arabic)

Alareeni, B. (2018). The impact of firm-specific characteristics on earnings management: evidence from GCC countries. International Journal of Managerial and Financial Accounting, 10(2), 85-104. https://doi.org/10.1504/IJMFA.2018.091659

Alareeni, B., \& Branson, J. (2013). Predicting Listed Companies' Failure in Jordan Using Altman Models: A Case Study. International Journal of Business and Management, 8(1), 113. https://doi.org/10.5539/ijbm.v8n1p113

Alareeni, B.A. (2019). The associations between audit firm attributes and audit qualityspecific indicators: A meta-analysis, Managerial Auditing Journal, 34(1), 6-43. https://doi.org/10.1108/MAJ-05-2017-1559

Al-Ghunaim, Muhammad. (2016). Billions of philanthropists are disabld, investigations and reports, Al-Riyadh newspaper, Saudi Arabia. (in Arabic)

Al-Harbi, Haiah Muhammad Saeed. (2002). Total quality management as an entrance to the development of Saudi universities, a study of the Saudi academy's attitudes toward the application of its principles, and their perspective on the contribution of this application to the development of the university, unpublished $\mathrm{PhD}$ thesis, Umm Al-Qura University, Makkah Al-Mukarramah. (in Arabic)

Al-Jarifani, Imad Abdullah (2008). Guiding quality in charitable work through the balanced scorecard, quality and charitable activities, the ninth annual meeting of charitable organizations in the eastern province, Saudi Arabia. (in Arabic)

Al-Jubouri, Jamal Majmoud. (2011). The impact of implementing total quality management on beneficiary services in non-governmental islamic relief organizations, Omdurman Islamic University, Sudan. (in Arabic)

Al-Orabi, Hamza, and Nawara Iman. (2016). The role of total quality management in strengthening corporate governance, Journal of Economic Research, (2), 146. (in Arabic)

Al-Otaibi, Faleh shugaa. (2010). The extent of commitment to the application of total quality management standards and their impact on the level of human resources performance an applied study on human resources management in the Kuwaiti National Guard, Kuwait: Middle East University. (in Arabic)

Al-Qurashi, Hussain bin Reda (2008). Quality standards for distinguished charitable work, harmonization of the standards of the King Abdulaziz Award for Quality for Charitable Work, Quality and Charitable Work, the ninth annual meeting of charities in the Eastern Province, Saudi Arabia. (in Arabic)

Al-Rabiah, Abdullah Muhammad; Shreim, Hassan Muhammad; And Al-Rasheed, Muhammad Nasser (2008). The extent of awareness of workers in charitable organizations in the Kingdom of Saudi Arabia to the concept of quality implementation, Quality and Charitable Work, the ninth annual meeting of charitable organizations in the Eastern Province, Saudi Arabia. (in Arabic)

Alsalahat, Sami. (2013). Endowment quality, a study in management and development standards, Journal of Sharia and Islamic Studies, Kuwait University, (28), 94. (in Arabic)

Al-Saleh, Mohammed bin Ahmed. (2001). Waqf in Islamic law and its impact on community development, Vol. 1, King Fahd National Library, Riyadh, Saudi Arabia. (in Arabic)

Alshaya, Mohammed Abdullah. (2013), Evaluating the level of total quality practice in charitable societies from the viewpoint of workers: a study applied to family 
associations in the cities of Buraidah and Al-Rass. Journal of Human Sciences, Majma'ah University, (3), 15-74. (in Arabic)

Al-Thubaiti, Zaban Bin Eida. (2008). Total quality in charitable work ... the beneficiary first, quality and charitable work, the ninth annual meeting in the Eastern Province, Saudi Arabia. (in Arabic)

Al-Thumairi, Abdul Salam. (2016) $.81 \%$ of endowment problems are court jurisprudence and mismanagement, News and Reports, Economic Newspaper, Riyadh, Saudi Arabia. (in Arabic)

Aqili, Omar Wasfi (2001). The integrated methodology for total quality management (point of view), Wael Publishing House, Amman, Jordan. (in Arabic)

Aumira, Mohammed bin Abdulaziz. (2003). Total quality relationship with functional performance in the health sector, Prince Sultan Center for Cardiac Treatment and Surgery for the Armed Forces in Riyadh, Saudi Arabia. (in Arabic)

Badr, Rashad Mahmoud. (2009). The impact of application of total quality principles on the performance of foreign ngos operating in the gaza strip, Master Thesis, Faculty of Commerce, Islamic University - Gaza, Palestine. (in Arabic)

Ben Ishaoui, Ahmed. (2008). Total quality management in hotel institutions in algeria, University of Algiers, Algeria. (in Arabic)

Bin Ishawi, Ahmed: (2006). Total quality management in service institutions, Algeria: Al-Baheth Journal, (4). (in Arabic)

Boresh, Hisham. (2017). Endowment institutions: between measuring strategic performance and improving the quality of their services, Prince Abdul Qadir University for Islamic Sciences, Jordan. (in Arabic)

Costandi, S., Hamdan, A., Alareeni, B., \& Hassan, A. (2019). Educational governance and challenges to universities in the Arabian Gulf region. Educational Philosophy and Theory, 51(1), 70-86. https://doi.org/10.1080/00131857.2018.1434621

Habashi, Fethiye. (2007). Total quality management with an applied study in the fermal pharmaceutical production unit of constantine, University of Mentouri Constantine, Algeria. (in Arabic)

Hakem, Adhem. (2017). Methods of Waqf administration, Master Thesis, Faculty of Law and Political Science, University of Dr. El-Taher Moulay-Saida, Algeria.

Hassan, Nubian Muhammad. (2011). Islamic endowment and urbanism, Vol. 1, King Saud University, Riyadh, Saudi Arabia. (in Arabic)

Hoffherr, G. e. (1994). Break-through thinking in total quality management Newjersey: Engle wood Cliffs.

Kharashi, Abdullah bin Othman. (2008). Quality management applications in charities. Quality and charitable work, the ninth annual meeting of charitable organizations in the Eastern Province, Saudi Arabia. (in Arabic)

Lashraf, Nassima. (2011). Total quality management standards as supporting the quality of hospital services, Qasid University. (in Arabic)

Ministry of Justice. (2019). Developing waqf work at the table of the "justice" and "labor" ministers. (in Arabic)

Qada, Yazid. (2012). The reality of the application of total quality management in Algerian educational institutions, Abi Bakr Belguid University, Algeria. (in Arabic)

Saleh, Amir Omar Hassanein. (2015). The impact of total quality management as a strategy for developing academic performance at taif university. Orient Journal for Scientific Studies and Research, (9). (in Arabic) 
Saudi Press Agency. (2019). Riyadh Chamber sheds light on the challenges of the endowment sector. www.spa.gov.sa/viewfullstory.php?lang=ar\&newsid=1984356 (access date 12 10, 2020). (in Arabic)

Saudi Quality Council. (2019): https://www.sqc.org.sa/index.php?option=com_content\&view=article\&id=114\&It emid=83\&lang=sa. (Accessed: 4/8/2020). (in Arabic)

Sheehani, Seham. (2010). Intervention entitled: The possibility of applying the total quality management system in higher education - the national forum on total quality management and institution performance development, Saida Yume University, Algeria. (in Arabic)

Sied Ahmed; Haji, Isa; And Tamimi, Wafa Sobhi Saleh. (2013). Application of total quality management to improve personnel performance, Blida University, Algeria. (in Arabic)

Zadira, Sharafuddin. (2017). The role of total quality management in improving the quality of services in hospital institutions, Mohamed Khaider University, Biskra, Algeria. (in Arabic)

Zakzouq, Khaled Jamil. (2008). Application of total quality management principles to improve the performance of the college of community service and continuing education at umm al-qura university, Umm Al-Qura University, Makkah AlMukarramah. (in Arabic) 